\title{
Millisecond Timescale Disinhibition Mediates Fast Information Transmission through an Avian Basal Ganglia Loop
}

\author{
Arthur Leblois, ${ }^{1}$ Ágnes L. Bodor, ${ }^{1}$ Abigail L. Person, ${ }^{3}$ and David J. Perkel ${ }^{1,2}$ \\ Departments of ${ }^{1}$ Otolaryngology and ${ }^{2}$ Biology and ${ }^{3}$ Graduate Program in Neurobiology and Behavior, University of Washington, Seattle, Washington 98195
}

\begin{abstract}
Avian song learning shares striking similarities with human speech acquisition and requires a basal ganglia (BG)-thalamo-cortical circuit. Information processing and transmission speed in the BG is thought to be limited by synaptic architecture of two serial inhibitory connections. Propagation speed may be critical in the avian BG circuit given the temporally precise control of musculature during vocalization. We used electrical stimulation of the cortical inputs to the BG to study, with fine time resolution, the functional connectivity within this network. We found that neurons in thalamic and cortical nuclei that are not directly connected with the stimulated area can respond to the stimulation with extremely short latencies. Through pharmacological manipulations, we trace this property back to the BG and show that the cortical stimulation triggers fast disinhibition of the thalamic neurons. Surprisingly, feedforward inhibition mediated by striatal inhibitory neurons onto BG output neurons sometimes precedes the monosynaptic excitatory drive from cortical afferents. The fast feedforward inhibition lengthens a single interspike interval in BG output neurons by just a few milliseconds. This short delay is sufficient to drive a strong, brief increase in firing probability in the target thalamic neurons, evoking short-latency responses. By blocking glutamate receptors in vivo, we show that thalamic responses do not appear to rely on excitatory drive, and we show in a theoretical model that they could be mediated by postinhibitory rebound properties. Such fast signaling through disinhibition and rebound may be a crucial specialization for learning of rapid and temporally precise motor acts such as vocal communication.
\end{abstract}

\section{Introduction}

The CNS provides several examples of circuits specialized for fast processing because they subserve tasks in which long transmission delays have important behavioral costs. For instance, auditory information reaches the forebrain through a polysynaptic pathway within $10 \mathrm{~ms}$ (Heil and Scheich, 1991; Sen et al., 2001; Pollak et al., 2003). Similarly the vestibulo-ocular reflex can cause eye movements within $10-15 \mathrm{~ms}$ of head motion (Broussard et al., 1992). Transmission speed is critical in neural systems involved in vocal communication in humans and birds because the duration of perceptually critical sounds in speech and bird songs can be as short as $10 \mathrm{~ms}$ (Liberman et al., 1961; Goller and Cooper, 2004; Glaze and Troyer, 2007).

The forebrain nuclei mediating song control form two segregated pathways (see Fig. 1A) (Nottebohm et al., 1976). The robust nucleus of the arcopallium (RA) receives input from the

\footnotetext{
Received June 26, 2009; revised Sept. 7, 2009; accepted Sept. 24, 2009.

This work was supported by National Institutes of Health Grant R01-MH066126 (D.J.P.) and a Grant Lavoisier (Ministère des Affaires Etrangères, France) (A.L.). We thank F. Rieke, G. Murphy, L. Acsády, D. Hansel, T. Boraud, and members of the Perkel laboratory for their comments on this manuscript. The GABA antibody for postembedding reaction was kindly donated by Dr. Peter Somogyi. We thank Yanping Huang and Alnur Ali for valuable help developing the model.

Correspondence should be addressed to Arthur Leblois, Department of Otolaryngology, University of Washington, 1959 NE Pacific Street, Box 356515, Seattle, WA 98195. E-mail: aleblois@u.washington.edu.

A. L. Person's present address: Department of Neurobiology and Physiology, Northwestern University, Evanston, IL 60208.

DOI:10.1523/JNEUROSCI.3060-09.2009

Copyright $\odot 2009$ Society for Neuroscience $\quad$ 0270-6474/09/2915420-14\$15.00/0
}

cortical (for discussion of nomenclature, see Materials and Methods) area HVC (proper name) both monosynaptically and polysynaptically, through a basal ganglia $(\mathrm{BG})$-thalamo-cortical circuit called the anterior forebrain pathway (AFP) (Bottjer et al., 1989). This BG pathway is essential for vocal learning (Bottjer et al., 1984; Scharff and Nottebohm, 1991). Song-related signals from HVC propagate through these two pathways (Kimpo et al., 2003), which converge on individual RA neurons (Mooney and Konishi, 1991). For the signals themselves to interact in RA, propagation delays should match.

Transmission delays within the BG, however, are expected to slow down signal propagation through the AFP. Indeed, activation of the thalamus through the BG first requires disinhibition, the interruption of the tonic inhibition exerted on thalamic neurons by pallidal neurons (Deniau and Chevalier, 1985). In mammals, latencies of $>20 \mathrm{~ms}$ have been reported between cortical activation and pallidal inhibition (Nambu et al., 2000). Additional delays could be introduced depending on the mechanisms driving thalamic neurons during disinhibition. In songbirds, postinhibitory rebound in thalamic neurons allows them to respond to disinhibitory signals in the absence of excitation (Luo and Perkel, 1999b; Person and Perkel, 2005). Although faster than in mammals (Thomson, 1988 ), postinhibitory rebound might impose additional delays $(>10 \mathrm{~ms})$ between the cortical drive and the thalamic response. Based on time lags in correlated activity in the song system, Kimpo et al. (2003) have hypothesized transmission delays through the AFP of $\sim 60 \mathrm{~ms}$. Troyer and Doupe (2000) 
proposed a theoretical framework allowing learning despite long AFP delays. However, shorter delays would support a wider range of possible learning mechanisms.

We used electrical stimulation to measure with high temporal precision the time course of information flow through the songbird BG circuit. We report surprisingly fast transmission through the AFP, with latencies as short as $10 \mathrm{~ms}$, relying on a BG disinhibitory circuit specialized for speed. AFP signals may thus overlap in time in RA with signals from the monosynaptic motor pathway, expanding the range of possible mechanisms underlying vocal learning.

\section{Materials and Methods}

Animals. Adult male zebra finches (Taeniopygia guttata) were obtained from a commercial supplier and used in accordance with an animal use protocol approved by the University of Washington Institutional Animal Care and Use Committee. Animals were housed under a 14/10 h light/ dark cycle with food and water available ad libitum.

Surgery. Animals were first food deprived for $30 \mathrm{~min}$ and were then given three intramuscular injections totaling 5-6.5 $\mathrm{ml} / \mathrm{kg} 20 \%$ urethane over $1 \mathrm{~h}$. Urethane anesthesia preserves normal neurotransmission in various subcortical areas and the peripheral nervous system (Maggi and Meli, 1986). Local anesthetic (1\% lidocaine) was injected under the scalp before animals were placed in a stereotaxic apparatus. Small craniotomies were made above the midline reference point, the bifurcation of the midsagittal sinus, and above HVC and the lateral magnocellular nucleus of the anterior neostriatum (LMAN), the medial portion of the dorsolateral nucleus of the anterior thalamus (DLM) or Area X unilaterally. Lidocaine gel was then applied to the incision at $3 \mathrm{~h}$ intervals.

Electrophysiological recording. Glass pipettes (TW100F-3; World Precision Instruments) were pulled on a micropipette puller (model P97; Sutter Instruments), and the tips were blunted to achieve 5-25 $\mathrm{M} \Omega$ impedance. A ground electrode was placed in the cerebellum posterior to the midline reference point. A concentric stimulation electrode (FHC) was placed in HVC $(0 \mathrm{~mm}$ rostral, $2.4 \mathrm{~mm}$ lateral from the midline reference point, $0.5 \mathrm{~mm}$ deep). The recording electrode signal was amplified $10 \times$ and low-pass filtered at $3 \mathrm{kHz}$ (Axoclamp2B amplifier; Molecular Devices), passed through a Hum Bug noise eliminator (AutoMate Scientific), and amplified further $100 \times$ (model 410; Brownlee Precision). Recordings were monitored using an oscilloscope and an audio monitor. We searched for single-unit neuronal activity in Area X, DLM, or LMAN using HVC stimulation as a search stimulus. Once a neuron was isolated, the electrophysiological signal was sampled at $20 \mathrm{kHz}$, and spike times and raw traces were stored for additional analysis (Spike2; Cambridge Electronic Design). Principal components analysis of the spike shapes allowed clear separation from noise, and all extracted units obeyed a refractory period of $1 \mathrm{~ms}$. In recordings in Area X, neurons displaying spontaneous firing above 25 spikes per second (sp/s) are referred to as pallidal neurons given their similarity with pallidal terminals recorded in DLM (Person and Perkel, 2007), whereas neurons displaying a firing rate lower than $25 \mathrm{sp} / \mathrm{s}$ are called putative interneurons. Recordings were performed during HVC microstimulation (monophasic $0.2 \mathrm{~ms}$ single pulses), with various stimulation intensities (10-4000 $\mu \mathrm{A})$. Each pulse of HVC microstimulation saturated the amplifier and occluded spiking activity for $1-2 \mathrm{~ms}$ in the recordings, as a result of the "overshoot" after saturation. Because the duration of this stimulation artifact was much shorter than the latency of the fastest responses recorded, it occluded only spontaneous activity and thus did not alter our analysis.

Previous studies have shown that stimulation with a monopolar microelectrode at $200 \mu \mathrm{A}$ activates $\sim 50 \%$ of the neurons located in a shell of $0.2 \mathrm{~mm}$ outside radius (Ranck, 1975; Tehovnik et al., 2006). These values should be considered with caution because the current intensity necessary to activate an axon at a given distance depends on a number of other variables such as the size of the axon or its biophysical properties (e.g., cellular excitability or axon myelination). In addition, concentric bipolar electrodes greatly reduce current spread, especially for higher stimulation intensities (Bagshaw and Evans, 1976; Follett and Mann, 1986), and estimates from monopolar electrodes thus provide an imprecise upper bound of the activated volume. The volume of the "activated shell" for $200 \mu \mathrm{A}$ (inside and outside radius: 0.1 and $0.2 \mathrm{~mm}$ ) is $0.029 \mathrm{~mm}^{3}$ and represents $\sim 10 \%$ or less of HVC volume $\left(0.2-0.5 \mathrm{~mm}^{3}\right.$ ) (MacDougallShackleton et al., 1998). Therefore, $200 \mu$ A pulses applied near the center of HVC through concentric bipolar electrodes are expected to activate $<5 \%$ of HVC neurons.

We cannot exclude the possibility that some high-amplitude stimulation led to current spread to neighboring structures. However, because of the segregation of the AFP circuit from surrounding tissue, it seems unlikely that occasional activation of neighboring structures would modify the interpretation of the present data.

Paired recordings. Pairs of units with distinct waveforms were recorded simultaneously at 14 DLM sites. These recordings always included a rapidly firing unit with a monophasic spike waveform of smaller amplitude and a more slowly firing unit with a biphasic waveform of larger amplitude. The waveforms differed in amplitude by at least fourfold, permitting unambiguous classification of spikes. These recordings have been shown to reflect the activity of a presynaptic pallidal terminal and the corresponding postsynaptic thalamic cell (Person and Perkel, 2007). In all cases, the extracted units obeyed a refractory period of $1 \mathrm{~ms}$. Because previous recordings of song-related and spontaneous activity gave similar results, they were pooled in the analysis.

Antidromic activation. We recorded high-frequency, spontaneously active neurons in Area X thought to be the pallidal projection neurons. We found no significant difference in the spike shapes of high versus low spontaneous activity neurons in Area X. The distributions of spontaneous activity between these neurons and terminals within DLM were not significantly different (see Fig. 4A) (73 Area X putative somata averaged $62.8 \pm 17.3 \mathrm{sp} / \mathrm{s} ; 37 \mathrm{DLM}$ terminals averaged $60.9 \pm 13.6 \mathrm{sp} / \mathrm{s})$. In some experiments, a parallel bipolar stimulating electrode $(0.5 \mathrm{~mm}$ spacing; FHC) was placed in DLM for antidromic identification of projection neurons. Continuous analog records were acquired for at least 300 stimuli, each of which consisted of single monophasic pulses delivered at $0.1 \mathrm{~s}$ intervals $(0.1 \mathrm{~ms}$ duration, $0.2-2 \mathrm{~mA}$ intensity, stimulus isolation unit from ISO-flex; A.M.P.I.). Latency variability was defined as the SD of latencies to the first evoked spike. Putative projection neurons were defined as having evoked spikes with small latency variability $(<0.2 \mathrm{~ms})$ and collision tests resulting in $100 \%$ failure of the antidromic spike.

Drug injections. All drugs were diluted in a $0.9 \%$ saline solution with 0.5\% dextran-conjugated Alexa-fluor 488 (3000 molecular weight; Invitrogen). The role of $\mathrm{GABA}_{\mathrm{A}}$ receptor-mediated synaptic transmission in Area $\mathrm{X}$ was examined using microinjection of the $\mathrm{GABA}_{\mathrm{A}}$ receptor blocker gabazine (0.1-0.2 mM; Tocris Bioscience). The role of glutamatergic transmission in Area X and DLM was examined using microinjection of the AMPA receptor blocker NBQX (1 mM; Tocris Bioscience) and the NMDA receptor blocker APV (5 mm; Tocris Bioscience) in $0.2 \%$ DMSO. Vehicle solution consisted of either saline or 0.2\% DMSO solution. Drugs were pressure ejected from pulled glass pipettes $(10-20 \mu \mathrm{m}$ tip size) using a Pressure system IIe (50 ms pulses at $10-16$ psi; Toohey). Injected volumes were $20-100 \mathrm{nl}$. When the recording and drug injections were made in the same structure, we aimed to place the tip of the injection pipette $200-300 \mu \mathrm{m}$ from the tip of the recording pipette.

Anatomy. At the end of each experiment, recording sites were labeled by iontophoretic injections of fluorescent dye [5\% Alexa-flour 488- or 568 -conjugated $10 \mathrm{kDa}$ dextran amine in $0.01 \mathrm{M}$ phosphate buffer (PB), $\mathrm{pH} 7.4$, ejected by $5 \mu \mathrm{A}$ alternating current for $5 \mathrm{~min}]$. Animals were killed by intramuscular injection of sodium pentobarbital (Nembutal) and perfused with $0.9 \%$ saline, followed by $4 \%$ paraformaldehyde as fixative. The brain was then removed, postfixed in $4 \%$ paraformaldehyde for $24 \mathrm{~h}$, and cryoprotected in $30 \%$ sucrose. Sections, $40 \mu \mathrm{m}$ thick, were then cut in the parasagittal plane on a freezing microtome and processed for histological examination to verify the location of stimulating and recording electrodes and drug injection sites. In addition to gross observation of electrode tracts, the brain slices were visualized using a fluorescence microscope allowing better determination of recording location. In the case of DLM recordings, descending axons from Area X were clearly retrogradely labeled in most cases, allowing unambiguous determination of recording site. We were able to recover dye deposits in DLM 
after recording in 13 animals (of 23 animals in which DLM recordings were conducted).

For quantification of Area X projection axon size, bilateral iontophoretic injections of biotinylated dextran amine (BDA) $(10,000$ molecular weight; $10 \%$ in $0.1 \mathrm{M} \mathrm{PB}, \mathrm{pH}$ 7.4; Invitrogen) were made into Area X. After a survival time, birds were killed with pentobarbital as described above and then perfused transcardially, first with physiological saline followed by fixative containing $2 \%$ paraformaldehyde (TAAB, UK) and $0.5 \%$ glutaraldehyde (TAAB) in acetate buffer, pH 6.0 (5 min), and finally with fixative containing $2 \%$ paraformaldehyde and $0.5 \%$ glutaraldehyde in borate buffer, $\mathrm{pH}$ 8.5 (50 min). Parasagittal sections $(60 \mu \mathrm{m})$ containing DLM and Area X were cut with a vibratome. After pretreatment of sections, injection sites and labeled fibers were visualized with a nickel-intensified 3,3'-diaminobenzidine (DABNi) reaction resulting in bluish-black reaction product [sections were first incubated with avidin biotinylated-horseradish peroxidase complex (ABC) (Vector Laboratories Burlingame, CA) and then developed with DABNi]. To examine the GABA content of the labeled axons, the tracer was visualized using a preembedding gold method followed by a postembedding anti-GABA reaction (Bodor et al., 2008). Briefly, sections were first incubated with $\mathrm{ABC}$ followed by a signal amplification step using biotinylated tyramide reagent (PerkinElmer Life and Analytical Sciences), incubated in $1 \mathrm{~nm}$ gold-conjugated streptavidin (Aurion), postfixed in $2 \%$ glutaraldehyde, and then silver intensified with Aurion R-Gent intensification kit. Light microscopic images were taken only from DAB-stained sections with a $100 \times$ oil-immersion objective.

Electron microscopy. DABNi and gold-labeled sections were treated with $\mathrm{OsO}_{4}$ and uranyl acetate, dehydrated in ethanol and propylene oxide, and embedded in Durcupan (ACM; Fluka). From DABNi and gold-labeled tissue $60 \mathrm{~nm}$ thick (silver color) ultrathin sections were cut, and sections were mounted on copper single slot grids. Finally, sections were stained with lead citrate and washed in distilled water. Postembedding GABA immunostaining was performed on gold-labeled tissue mounted on nickel grids according to Somogyi and Hodgson (1985). Electron micrographs were taken using a camera (Morada; Olympus) connected to an electron microscope (JEM 1200 EXII; JEOL). In Figure 4D, the tracer BDA was visualized with preembedding streptavidin gold labeling. The ultra-small gold particles were silver intensified (larger magnification of the area indicated by the black square shown in inset). This method reveals morphological details of the large axons, such as the structure of the filaments and myelin.

Data analysis. Spike times were analyzed using Matlab 7.0.1 software (MathWorks). For each cell, we calculated spontaneous firing rate, interspike interval (ISI) distribution, and peristimulus time histogram (PSTH) of the response to HVC stimulation. Whereas the PSTHs displayed in Figures 1, 2, 5, and 7 have a $1 \mathrm{~ms}$ bin and are not smoothed, additional analyses were performed on PSTHs smoothed as follows. For each trial, the firing rate time course was determined with $1 \mathrm{~ms}$ resolution by convolving the spike train with a Gaussian kernel of width $1 \mathrm{~ms}$ (Baker and Gerstein, 2001). The mean and SD of the spontaneous rate were determined over the $100 \mathrm{~ms}$ preceding stimuli. A neuron was considered to display a significant response if at least two consecutive bins of the PSTH were beyond limits defined by the spontaneous mean $\pm 2.5 \mathrm{SD}$. Responses were often made up of several components (especially in Area $\mathrm{X}$ pallidal cells), some inhibitory and some excitatory. We defined the beginning of the response component as the time of the first of two

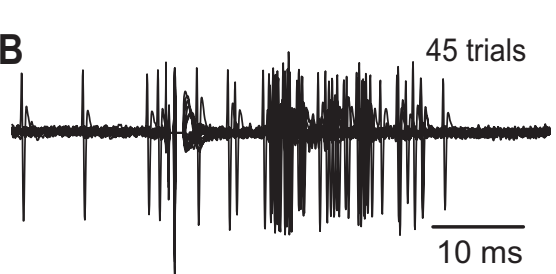

B

C

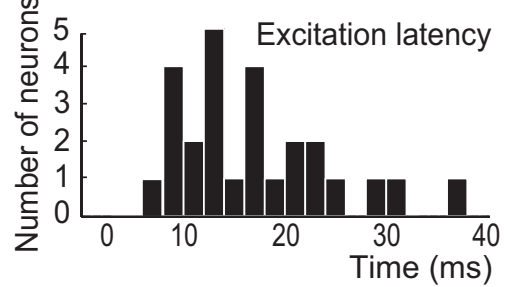

Time (ms)

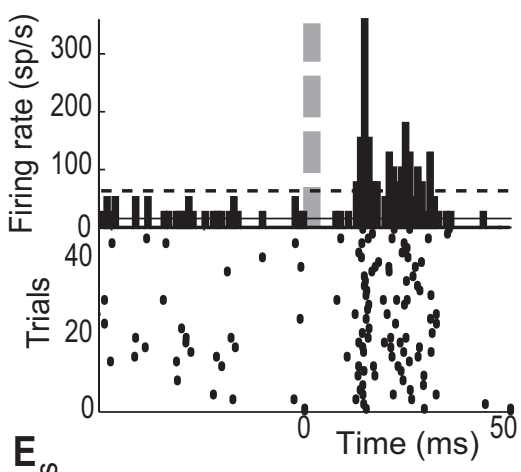

Time (ms)

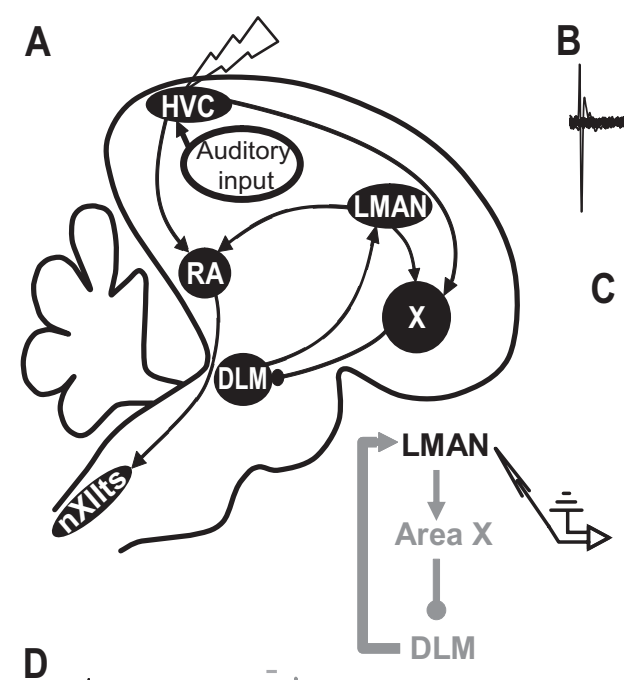

1. The response of LMAN neurons to HVC electrical stimulation. $A$, Schematic parasagittal representation of the

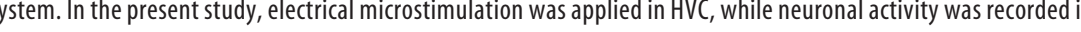
uron in LMAN surrounding HVC stimulation. C, PSTH (top; horizontal solid line denotes average spontaneous firing rate; for the neuron shown in $\boldsymbol{B}$ and the corresponding raster plot (bottom; 45 stimuli). $\boldsymbol{D}$, Average PSTH (solid black line) around HVC stimulation over all LMAN neurons $(n=26)$, with confidence limits (gray lines; mean \pm SEM). Horizontal dashed black line indicates the average spontaneous firing rate. $E$, Distribution of excitation latency in LMAN after HVC urons displayed response latencies $<20 \mathrm{~ms}$.

consecutive bins of the PSTH in which the firing rate fell outside significance limits; similarly, the end occurred when two consecutive bins fell back within significance limits. In DLM and LMAN, the response latency was defined as the latency to the first component of the response, whereas response duration was summed over all components. For pallidal neurons, we defined the latency to excitatory or inhibitory components as the latency to the first such component. The duration of excitatory or inhibitory components was the sum of the duration over all such components, respectively. Response strength was calculated over a response window as follows. First, the area of the PSTH above (or below) baseline firing rate was calculated as the sum of the differences between the PSTH bins above (or below) baseline firing and the mean baseline firing rate. These areas were then divided by the population average spontaneous firing rate multiplied by the length of the response window (35 $\mathrm{ms}$ in Area X, $60 \mathrm{~ms}$ in DLM). The result was called excitation (or inhibition) strength and expressed as a percentage of baseline firing. For DLM neurons, Area X putative interneurons and pallidal neurons displaying only excitatory responses, the lowest stimulation current intensity evoking a reliable response (at least one additional spike in each trial), was selected for additional analysis. For pallidal neurons displaying some inhibition in response to stimulation, the lowest current intensity evoking an inhibitory component in their response was selected for additional analysis. For responses in DLM neurons, the probability of response was calculated as the probability that a given trial contained at least one spike between 0 and $60 \mathrm{~ms}$ after HVC stimulation. The jitter in the response was defined as the SD of the time of the first spike in this window. For paired recordings in DLM, we computed the distribution of time lags between each thalamic spike and the immediately preceding pallidal spike. 


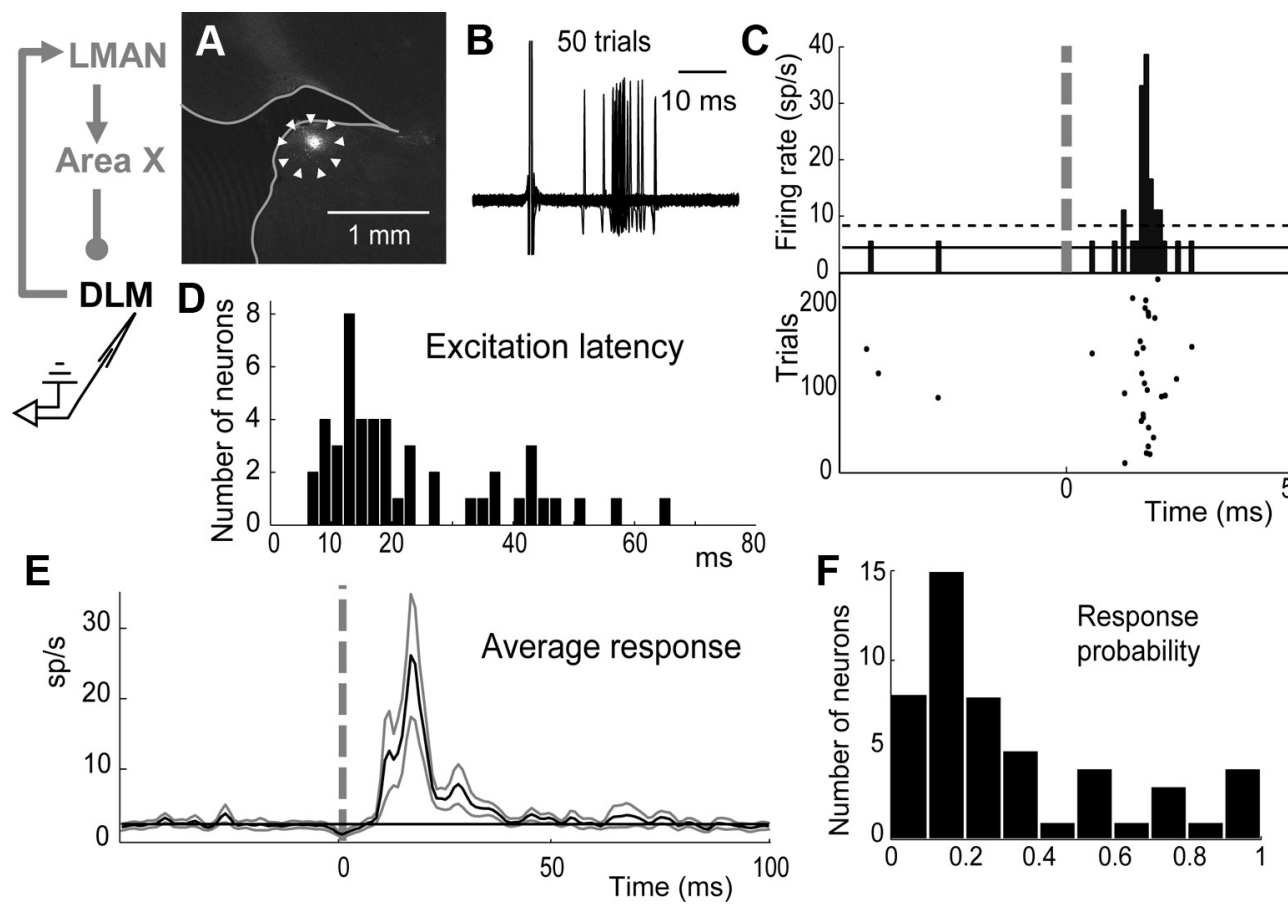

Figure 2. The response of DLM neurons to HVC electrical stimulation. $A$, Example of DLM labeling after dye injection at the end of an experiment. Contours of the brain are outlined with a thick white line, and triangles denote DLM boundaries. B, Superimposed raw traces (50) of a DLM neuron surrounding HVC stimulation. C, PSTH (top; horizontal solid line denotes average spontaneous firing rate; horizontal dashed lines depict mean $\pm 2.5 \mathrm{SD}$ ) around HVC stimulation (at $t=0$ ) for the DLM neuron shown in $\boldsymbol{B}$ and the corresponding raster plot (bottom; 220 stimuli). $\boldsymbol{D}$, Distribution of excitation latency in DLM after HVC stimulation ( $n=49$ ). Most neurons displayed response latencies $<20 \mathrm{~ms}$. $E$, Average PSTH (solid black line) for HVC stimulation (at $t=0$ ) for all 50 DLM neurons, with confidence limits (gray lines, mean \pm SEM). Horizontal black line indicates the average spontaneous firing rate. $F$, Probability of response among DLM neurons.
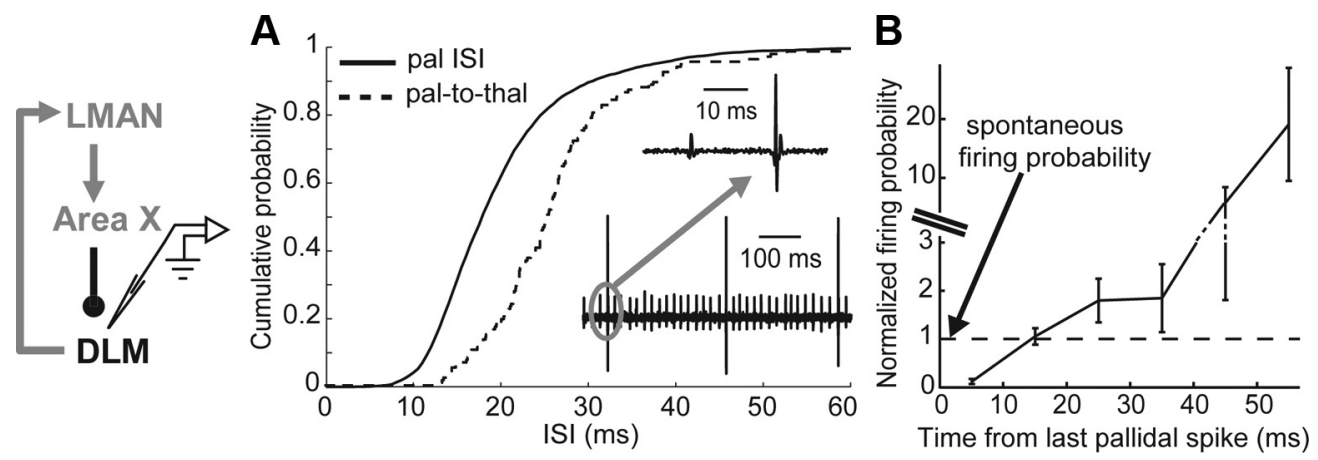

Figure 3. Relation between firing and pallidal input activity in DLM neurons: $A$, Cumulative distributions of the presynaptic pallidal terminal ISI (pal ISI, solid line; $n=6474)$ and the time intervals between postsynaptic spikes and the preceding presynaptic spike (pal-to-thal, dashed line; $n=132$ ) in a paired recording in DLM of a presynaptic pallidal terminal and the corresponding postsynaptic cell. Note the relatively long time between presynaptic and postsynaptic spikes compared with presynaptic ISIs. Bottom right inset, Example raw trace of this paired recording (bottom), with a zoomed-in $30 \mathrm{~ms}$ trace (top) showing two (smaller) presynaptic spikes and a (larger) postsynaptic spike, which typically occurred at the very end of the presynaptic ISI. $\boldsymbol{B}$, Average normalized firing probability of DLM neurons as a function of the time of the last spike of the presynaptic pallidal terminal. For each DLM cell and time bin, normalized probability was calculated as the firing probability in this bin divided by the firing probability in a time bin of the same size taken at random times.

Statistics. Numerical values are given as mean \pm SD, unless stated otherwise. Response latency, strength, and duration before and after drug injections were compared using a paired $t$ test. In addition, for each cell, spontaneous activity over multiple trials was compared before and after drug injection using a paired $t$ test. Axon-size measurements were made with NIH IMAGE J 1.40g, and datasets were compared using a $t$ test.

DLM neuron model. We built a single compartment conductancebased model of a DLM neuron using the NEURON simulation package (Hines, 1998). The parameters were based on the thalamic relay neuron models of McCormick and Huguenard (1992) and Destexhe et al. (1996). Channel conductances were tuned by hand such that model responses to current injection resembled those recorded (Luo and Perkel, 1999b). Soma diameter was $15 \mu \mathrm{m}$. Parameters for voltage- dependent $\mathrm{Na}$ and $\mathrm{K}$ currents were unchanged from the model of Destexhe et al. (1996), except that "vtraub" was $-45 \mathrm{mV}$. Maximal conductance density was $3 \mathrm{mS} / \mathrm{cm}^{2}$ for $\mathrm{Na}$ and $5 \mathrm{mS} / \mathrm{cm}^{2}$ for $\mathrm{K}$. Parameters for low-threshold $\mathrm{Ca}$ and $\mathrm{H}$ currents followed Destexhe et al. (1996), except that $\mathrm{Ca}$ dynamics were excluded and $\mathrm{H}$ current voltage dependence and kinetics were adjusted to match data of Luo and Perkel (2002). Maximal conductance for Ca was $4 \mathrm{mS} / \mathrm{cm}^{2}$ and for $\mathrm{H}$ current was $0.1 \mathrm{mS} / \mathrm{cm}^{2}$.

Intrinsic properties were then fixed, and a single inhibitory synaptic input was added. Reversal potential was set at $-95 \mathrm{mV}$ (Person and Perkel, 2005). The conductance waveform was the difference between two exponentials, in the form

$$
g_{\text {syn }}(t)=G^{*} e^{\left(-t / \tau_{\text {decay }}\right)}-e^{\left(-t / \tau_{\text {rise }}\right)}
$$


where $\tau_{\text {rise }}$ was $1.3 \mathrm{~ms}$ and $\tau_{\text {decay }}$ was $9 \mathrm{~ms}$ (Luo and Perkel, 2002). $G$ was set to give a peak conductance of $12.8 \mathrm{nS}$ (Luo and Perkel, 2002). No short-term synaptic plasticity was included.

Note concerning nomenclature. According to the revised nomenclature of the avian brain (Reiner et al., 2004b; Jarvis et al., 2005), nuclei HVC and LMAN lie in the avian pallium, the structure overlying the BG. Mammalian cortex is also a pallial structure, along with the amygdala and claustrum. Although there remains some controversy regarding the precise evolutionary relationship between mammalian cortex and these avian pallial structures, we refer here to HVC and LMAN as "cortical." In addition, we refer to the spontaneously active output neurons of Area X as "pallidal cells" because they share many features with mammalian pallidal neurons (Farries et al., 2002). It is important to note that they also differ in some respects from mammalian pallidal neurons (Carrillo and Doupe, 2004; Gale et al., 2009). Such simplified nomenclature is intended to make this article more accessible to researchers interested in BG physiology but unfamiliar with the avian anatomy.

\section{Results}

LMAN and DLM neurons respond with very short latency to HVC stimulation

To measure propagation speed through the AFP, we made extracellular single-unit recordings from 26 neurons in LMAN and 50 neurons in DLM displaying a response to electrical stimulation of cortical nucleus HVC.

In both nuclei, spontaneous activity was low $(4 \pm 6 \mathrm{sp} / \mathrm{s}$ in LMAN and $2 \pm 3$ $\mathrm{sp} / \mathrm{s}$ in DLM) and irregular when it was present at all. The coefficient of variation (CV) of ISIs was $1.3 \pm 0.5$ in 26 of 26 LMAN neurons and $0.9 \pm 0.4$ in 36 of 50 DLM neurons. HVC stimulation evoked increases in firing in all LMAN neurons (Fig. $1 B, C$ ). The minimal stimulation current necessary to evoke responses in LMAN was $200 \mu \mathrm{A}$. The latency of these responses was surprisingly short in most neurons (Fig. $1 E$ ), with a median latency of $16 \mathrm{~ms}$. As a result, the population average PSTH undergoes a sharp rise starting at $11 \mathrm{~ms}$ and peaks at $17 \mathrm{~ms}$ after HVC stimulation (Fig. 1D). Firing irregularity remained high during the responses, with a CV of firing of $1.3 \pm 0.5$ in the $0-500 \mathrm{~ms}$ window after stimulation. Responses were usually reliable; the response probability was $0.6 \pm 0.3$ (range, $0.2-1$ ). The timing of these responses was both precise and brief, with a $9 \pm 5 \mathrm{~ms}$ jitter in the time of the first evoked spike. The response duration, defined as the number of consecutive bins beyond significance in the PSTH (see Materials and Methods), was $27 \pm 16 \mathrm{~ms}$. Although latency to LMAN responses informed us about the overall transmission delays in the AFP, we decided to investigate DLM responses to reveal the mechanisms allowing fast signal propagation through the BG.
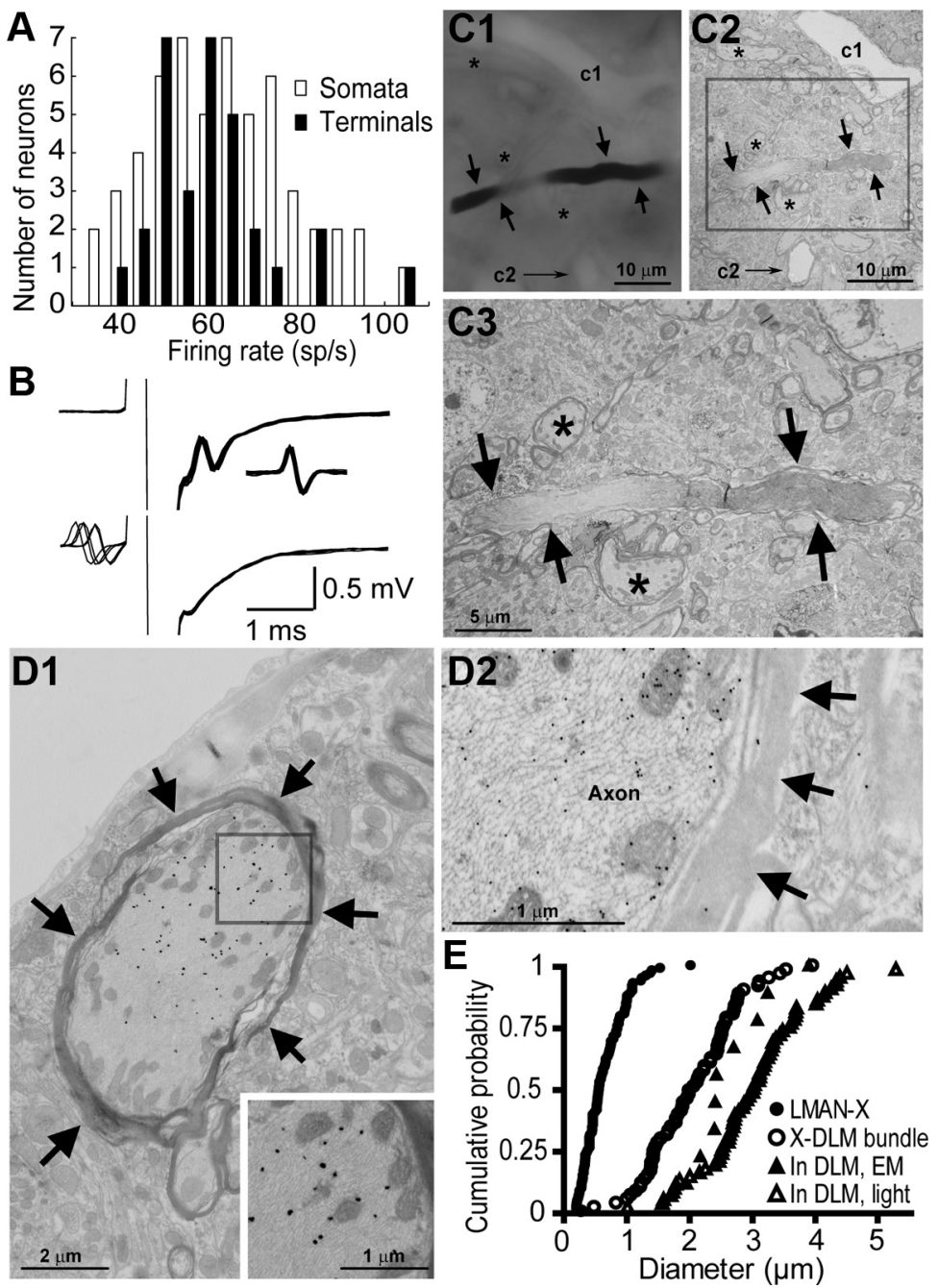

Figure 4. Comparison of Area $X$ output neuron somata and terminals and the morphological properties of their axons. $\boldsymbol{A}$, Distribution of spontaneous firing rates among pallidal neurons recorded in Area X (somata, white) or in DLM (terminals, black). Mean rate did not differ significantly. $\boldsymbol{B}$, Top, Superimposed traces (20 trials) of antidromic spike evoked by DLM stimulation in a pallidal cell recorded in Area X. The bottom right inset depicts superimposed traces (20 trials) of spontaneous spikes from the same neuron. Bottom, Superimposed traces (5 trials) of collision tests revealing a lack of antidromic activation when stimulation immediately followed a somatic spike. $\boldsymbol{C}$, Correlated light (C) and electron micrograph ( $\mathbf{C}$, magnified in $\mathbf{C}$ ) of the same anterogradely labeled axon (thick arrow) in DLM. In C1 and $\mathbf{C 2}$, other myelinated axons (asterisks) and capillaries ( 1 and $\mathrm{C} 2$ ) were used as landmarks to match the light and electron micrographs. D1, Electron micrograph of an anterogradely labeled axon in DLM showing the large size of the axon and revealing morphological details such as the structure of the filaments and myelin. Inset, Expanded view of the region surrounded by the black square. D2, Part of the same axon as in D1 after postembedding GABA immunolabeling. Small black dots are gold particles indicating the presence of GABA immunoreactivity. The preembedding anterograde label is absent because of the etching procedure used for GABA staining. $\boldsymbol{E}$, Cumulative histogram of the diameters of anterogradely labeled axons from Area X to DLM. The axon diameters were measured in the bundle just rostral to DLM (open circle) as well as in DLM (open triangles) at the light level after DABNi visualization and, in nine cases, at the electron microscopic level (filled triangles; 6 were correlated light and electron microscopic measurements and 3 were gold labeled). The filled circles represent retrogradely labeled axons between Area X and LMAN after the same BDA injections in Area X.

HVC stimulation also evoked rapid increases in firing in all but one DLM neurons (Fig. $2 B, C$ ). The minimal stimulation necessary to evoke responses in DLM was $100 \mu \mathrm{A}$. The median latency of these responses was $17 \mathrm{~ms}$ (Fig. 2D), and the population average PSTH rose at $10 \mathrm{~ms}$ and peaked at $18 \mathrm{~ms}$ after stimulation (Fig. 2E). The similarity in the median latency in LMAN and DLM is surprising at first glance. However, given the convergence of synaptic inputs from many DLM neurons to a single LMAN neuron, each LMAN neuron is expected to receive input from a pool of DLM neurons. In response to HVC stimulation, the DLM neurons displaying short latencies might drive fast re- 

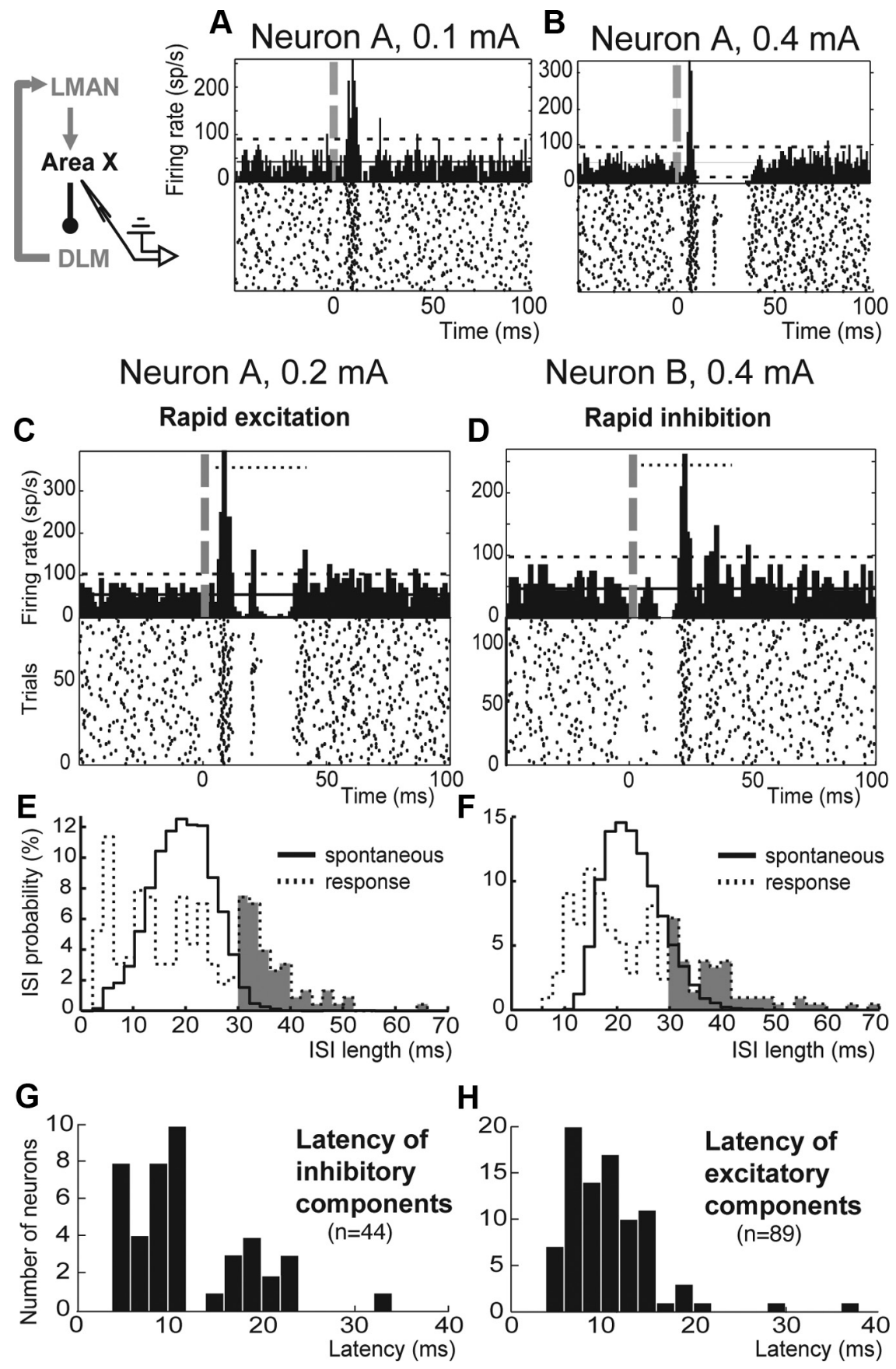

Figure 5. Response of pallidal neurons to HVC electrical stimulation. $A, B$, Responses of a pallidal neuron (Neuron $A$ ) to HVC electrical stimulation with two different stimulation intensities $(\boldsymbol{A}, 0.1 \mathrm{~mA} ; \boldsymbol{B}, 0.4 \mathrm{~mA})$. Each panel displays the PSTH (top; horizontal solid line denotes average spontaneous firing rate; horizontal dashed lines depict mean \pm 2.5 SD) and raster plot (bottom) of the response aligned to HVC stimulation. Low intensity stimulation $(\boldsymbol{A})$ evokes only excitation, whereas inhibition appears at higher stimulation intensities $(\boldsymbol{B})$. $\boldsymbol{C}$, The response of Neuron $A$ to the lowest stimulation current evoking inhibition ( $0.2 \mathrm{~mA}$, selected for additional analysis) consists of a rapid excitation followed by inhibition. The PSTH (top; horizontal solid line denotes average spontaneous firing rate; horizontal dashed lines depict mean \pm 2.5 SD) and its raster plot (bottom; 89 trials) are aligned to HVC stimulation. Dotted horizontal bars near the top of the PSTH panels in $\boldsymbol{C}$ and $\boldsymbol{D}$ indicate the window during which ISIs were measured for $\boldsymbol{E}$ and $\boldsymbol{F}$. $\boldsymbol{D}$, Example of a pallidal neuron displaying rapid inhibition preceding excitation in response to HVC stimulation. PSTH aligned to HVC stimulation (top; same conventions as in $\boldsymbol{A}$ ) with its raster plot below (bottom; 112 trials). $\boldsymbol{E}, \boldsymbol{F}$, Distribution of ISIs in spontaneous activity (black) and in response to HVC stimulation (dotted line; for ISIs overlapping with a 5- $40 \mathrm{~ms}$ window after stimulation) for the pallidal neuron depicted in $\boldsymbol{C}(\boldsymbol{E})$ and $\boldsymbol{D}(\boldsymbol{F})$. The contribution of ISIs longer than $30 \mathrm{~ms}$ in response to HVC stimulation is shaded in gray. $\boldsymbol{G}, \boldsymbol{H}$, Latency distribution of the inhibitory $(\boldsymbol{G})$ and excitatory $(\boldsymbol{H})$ components of responses to HVC stimulation in pallidal neurons. sponses in most LMAN neurons. Although DLM responses consisting of single spikes or doublets were stable and consistent over time, they were usually not elicited on each trial. The probability of response among the recorded neurons averaged $0.3 \pm 0.3$ (range, $0.04-1$ ) (Fig. $2 F)$, and the firing variability thus remained high in response to HVC stimulation (ISI CV of $0.8 \pm 0.3$ ). However, the timing of these responses, when elicited, was both precise and brief, with a $10 \pm 6 \mathrm{~ms}$ jitter in the time of the first evoked spike. The response duration, defined as the number of consecutive bins beyond significance in the PSTH (see Materials and Methods), was only $10 \pm 10 \mathrm{~ms}$.

In summary, despite low response probability, DLM neurons display fast and precise responses to HVC stimulation. The response latencies in LMAN and DLM are shorter than were anticipated given the number of synaptic connections between HVC and DLM and the disinhibitory mechanism involved.

Thalamic firing is dominated by the inhibitory pallidal input

The main input to DLM is GABAergic and arises from the BG (Bottjer et al., 1989; Luo and Perkel, 1999a; Person et al., 2008). Each DLM neuron receives input from a single or, at most, two large, aspiny Area X neurons (Okuhata and Saito, 1987; Luo and Perkel, 1999a). These neurons display high spontaneous firing rates and are termed pallidal neurons because of their resemblance to neurons in the mammalian pallidum (Farries et al., 2002; Reiner et al., 2004a). The large one-to-one calyxlike terminals formed by Area X pallidal neurons around DLM somata strongly hyperpolarize DLM neurons (Luo and Perkel, 1999b; Person and Perkel, 2005). Moreover, this configuration allows simultaneous recording of pairs of the presynaptic pallidal and postsynaptic thalamic units (Person and Perkel, 2007), and we recorded the activity in 14 such pairs. The spike times of thalamic neurons in these pairs were very strongly constrained by the firing pattern of their presynaptic pallidal terminals. Indeed, in 11 of 14 pairs, at least $90 \%$ of the thalamic spikes occurred $>10 \mathrm{~ms}$ after the preceding pallidal spike (Fig. 3A). As a result, the probability of firing of thalamic neurons was very low 0-10 ms after a pallidal spike. Their firing probability increased dramatically for longer delays after the last pallidal spike (Fig. $3 B$ ). This suggests that a thalamic neuron could fire only if the presynaptic pallidal ISI was long enough. 


\section{Most pallidal neurons project to the thalamus and have very short conduction latency}

Because the activity of DLM cells is dominated by their inhibitory somatic input from a single pallidal neuron, we investigated how short-latency responses to HVC stimulation in DLM could be mediated by pallidal neurons. We first measured the time it takes action potentials to propagate between Area X and DLM. We recorded from pallidal neurons in Area X (see Materials and Methods and Fig. $4 \mathrm{~A}$ for a comparison with terminals in DLM) and antidromically stimulated in DLM (Fig. $4 B$ ). We found that $67 \%$ (20 of 30) of the high-frequency spontaneously active neurons in Area X displayed antidromic activation in response to DLM stimulation, with a latency of $0.85 \pm 0.24 \mathrm{~ms}$ (latency variability of $62 \pm 30 \mu \mathrm{s})$. In contrast, axons from HVC to Area X have conduction latency $\sim 5 \mathrm{~ms}$ (Hahnloser et al., 2006) over a similar distance as that from Area X to DLM. In summary, at least two-thirds of Area X pallidal neurons appear to send an axon to DLM. Moreover, the axons from Area X to DLM conduct rapidly.

\section{Pallidal projection neurons have unusually large axons}

Consistent with the short conduction delays observed between Area $\mathrm{X}$ and DLM, the diameter of these axons was very large (Fig. 4). Light microscopy of axons anterogradely labeled from Area $X$ revealed diameters of $2.1 \pm 0.7 \mu \mathrm{m}$ as the axons entered DLM. Within DLM, axon diameters became even larger. Light and electron microscopy (EM) revealed diameters $>2.5 \mu \mathrm{m}$ (light, $3.0 \pm$ $0.8 \mu \mathrm{m}, n=63$; EM, $2.7 \pm 0.6 \mu \mathrm{m}, n=9)$. In addition, these large Area $\mathrm{X}$ axons remain myelinated in DLM (Fig. 4C,D). These large diameters contrast with other axons connecting song-system nuclei. Retrogradely labeled axons from LMAN to Area X, from the same injections used to label axons projecting to DLM, had significantly smaller diameters of $0.68 \pm 0.33 \mu \mathrm{m}(p<0.001, n=97)$. Comparing the conduction velocity of Area X-to-DLM neurons with large axons to smaller gauge axons between LMAN or HVC and Area X (LMAN, Fig. 4E; HVC, our unpublished observation) suggests that the large diameter of these axons saves at least $3 \mathrm{~ms}$, or $20 \%$ of the transmission delay from HVC to LMAN.

\section{Pallidal neurons display fast inhibition in response to HVC stimulation}

To understand how much time processing within Area X contributes to propagation delays between HVC and DLM, we investigated the response of pallidal neurons in Area X to HVC electrical stimulation. As expected, pallidal neurons recorded at the soma in Area X or the terminal in DLM exhibited rapid spontaneous activity $(62.4 \pm 15.6 \mathrm{sp} / \mathrm{s}, n=110)$, which, unlike DLM firing, was quite regular (ISI CV of $0.38 \pm 0.16$ ).

In response to low intensity stimulation in $\operatorname{HVC}(40-100 \mu \mathrm{A}$, $0.2 \mathrm{~ms}$ pulses), pallidal neurons displayed short-duration excitation, with a latency of $8.0 \pm 2.3 \mathrm{~ms}$ and a duration of $8.6 \pm 4.6 \mathrm{~ms}$ (Fig. 5A). Note that such low stimulation intensities usually did not evoke any response in DLM. When stimulation intensity was increased $(>100 \mu \mathrm{A})$, excitation became stronger, and, in many neurons, an inhibitory effect of stimulation appeared (Fig. $5 B-$ $D)$. The minimal stimulation current evoking feedforward inhibition in Area X pallidal cells was $200 \mu$ A. Surprisingly, inhibition was sometimes fast and often appeared before excitation (in $48.8 \%$ of the neurons displaying inhibition) (Fig. 5D). Shortduration excitation is likely mediated by monosynaptic connections from HVC, whereas inhibition is likely disynaptic, and observing the inhibitory response before excitation was thus unexpected. Such responses were recorded in pallidal neurons recorded in either Area X (somata) or DLM (terminals). Because
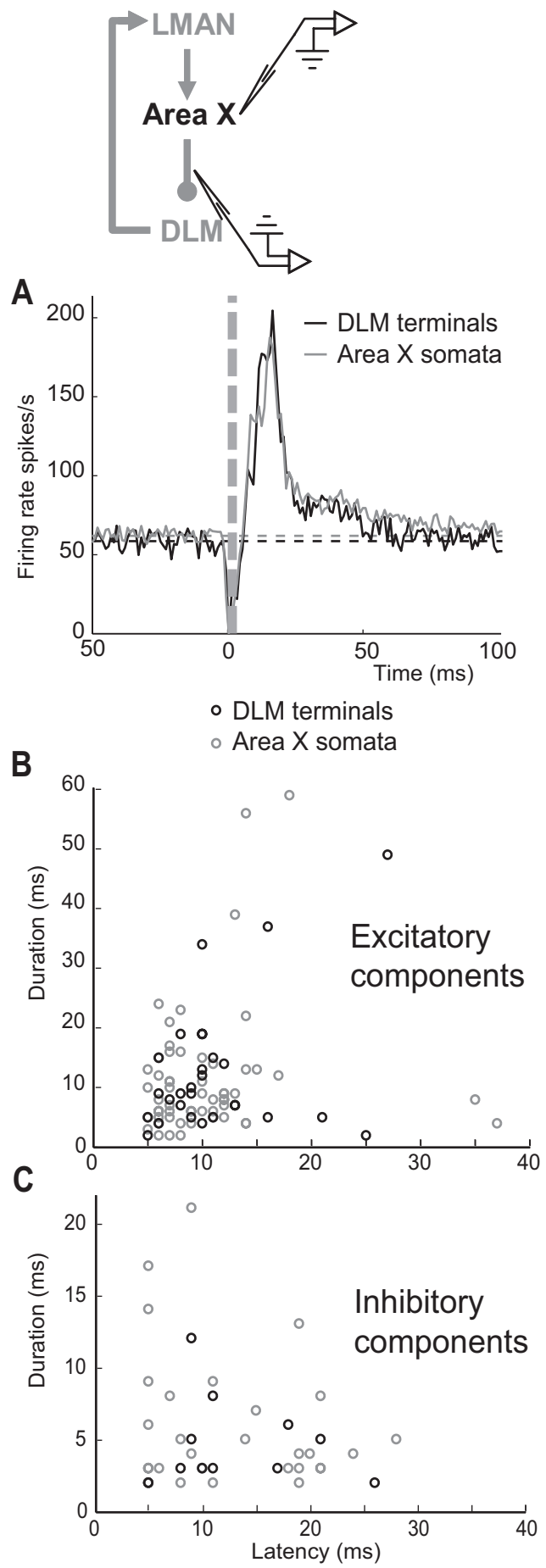

Figure 6. Responses to HVC electrical stimulation in pallidal neurons recorded in DLM (terminals) and Area X (somata). $A$, Average PSTH of the response to HVC stimulation in pallidal terminals in DLM (black) and pallidal cell somata in Area X (gray). Horizontal dashed lines depict average spontaneous activity in DLM terminals (black) and Area X cell somata (gray). $\boldsymbol{B}, \boldsymbol{C}$, Distribution of latency versus duration of the excitatory $(\boldsymbol{B})$ and inhibitory $(\boldsymbol{C})$ components of the responses to HVC stimulation in pallidal neurons recorded in DLM (terminals, black) and in Area $X$ (somata, gray). Because responses of pallidal neurons recorded in Area X (somata) or in DLM (terminals) were very similar, we pooled these two groups for the analysis of pallidal response to HVC stimulation.

responses were very similar in both groups (Fig. 6, Table 1), we pooled the data for additional analysis. As a result of combined excitatory and inhibitory effects, the first excitatory component of the pallidal neuron response had broadly distributed latencies 
Table 1. Fraction of neurons displaying excitation and inhibition in pallidal neurons recorded in DLM (terminals) and Area X (somata)

\begin{tabular}{llllll}
\hline & Excited only & Inhibited only & Inhibited-excited & Excited-inhibited & All \\
\hline DLM terminals & $17(53.1 \%)$ & 0 & $5(15.6 \%)$ & $10(31.3 \%)$ & 32 \\
Area X somata & $29(50 \%)$ & $1(1.7 \%)$ & $12(20.7 \%)$ & $16(27.6 \%)$ & 58 \\
All pallidal cells & $46(51.1 \%)$ & $1(1.1 \%)$ & $22(24.4 \%)$ & $21(23.3 \%)$ & 90 \\
\hline
\end{tabular}

Table 2. Response pattern to HVC electrical stimulation in pallidal neurons

\begin{tabular}{lllll}
\hline & $\begin{array}{l}\text { First } \\
\text { excitation }\end{array}$ & $\begin{array}{l}\text { First } \\
\text { inhibition }\end{array}$ & $\begin{array}{l}\text { Second } \\
\text { excitation }\end{array}$ & $\begin{array}{l}\text { Second } \\
\text { inhibition }\end{array}$ \\
\hline Number (fraction) & $89(98.9 \%)$ & $44(48.9 \%)$ & $53(58.2 \%)$ & $9(9.9 \%)$ \\
Latency (ms) & $10.4 \pm 5.6$ & $12.7 \pm 6.5$ & $32.5 \pm 16.2$ & $42.7 \pm 25.4$ \\
Duration (ms) & $11.1 \pm 11.1$ & $5.8 \pm 9.1$ & $9.9 \pm 14.0$ & $3.3 \pm 2.9$ \\
\hline
\end{tabular}

Values are shown as mean $\pm S D$. Approximately half of the recorded neurons displayed only excitation in response to HVC stimulation, whereas the other half displayed excitation followed or preceded by inhibition.

(median of $10 \mathrm{~ms}$, range of $4-25 \mathrm{~ms}, n=89$ ) (Fig. $5 H$, Table 2), and the latency to the early inhibitory component was just as short (median of $10 \mathrm{~ms}$, range of 5-28 ms, $n=44$ ) (Fig. 5G, Table 2).

Interestingly, relatively high stimulation intensities $(>100$ $\mu \mathrm{A})$ are necessary to evoke inhibitory components in the responses of pallidal neurons and to induce responses in downstream nuclei. Such intensities might be necessary to activate simultaneously a physiologically relevant number of neurons in HVC. Indeed, we estimated that a stimulation current of $200 \mu \mathrm{A}$ (sufficient to evoke an inhibitory component in many pallidal neurons) applied in the center of HVC is activating at most $5 \%$ of all HVC neurons (see Materials and Methods). Because neurons projecting to RA or X and interneurons are distributed homogeneously over the nucleus (Wild et al., 2005), we expect such stimulation to activate $5 \%$ of $\mathrm{HVC}$ neurons projecting to Area X $\left(\mathrm{HVC}_{\mathrm{X}}\right)$. During singing, almost all $(80 \%) \mathrm{HVC}_{\mathrm{X}}$ neurons fire one to four bursts of $6 \mathrm{~ms}$ average duration during each song motif (Kozhevnikov and Fee, 2007), corresponding to an average activation period of $10-20 \mathrm{~ms}$, or $2-4 \%$ of the duration of the motif $(\sim 500 \mathrm{~ms})$. If all $\mathrm{HVC}_{\mathrm{X}}$ neurons were firing independently, $2-4 \%$ of them would thus be simultaneously activated at any given time point in the song. As is apparent in the study by Kozhevnikov and Fee (2007, their Fig. 2 A), singing-related activity bursts seem often to happen simultaneously in 2-3 out of the $24 \mathrm{HVC}_{\mathrm{X}}$ neurons recorded in that bird. The ratio of simultaneously activated $\mathrm{HVC}_{\mathrm{X}}$ neurons might thus be higher than our estimate (closer to $10 \%$ ), at least at certain times during singing. The strong synchronized drive sent from HVC to the $\mathrm{BG}$ after electrical stimulation with high current $(>100 \mu \mathrm{A})$ thus seems to involve a similar number of HVC neurons as singing-related activation.

We then examined the distribution of the first few (two to three) ISIs evoked by HVC stimulation in pallidal neurons displaying an inhibitory component in their response. Their distribution extended over a range similar to that of ISIs evoked by song playback in those neurons (Person and Perkel, 2007), confirming that HVC electrical stimulation evoked behaviorally relevant activation patterns in these neurons. Because of an increased number of shorter ISIs after stimulation, the median ISI duration was shorter in response to HVC stimulation than at baseline ( $13 \pm 6$ vs $17 \pm 5 \mathrm{~ms}$ ). However, longer ISIs (>30 ms) were also more likely to occur after the stimulation than during episodes of spontaneous activity $(11.8 \pm 14.0$ vs $7.3 \pm 11.8 \%, p=$ 0.03 ) (Fig. $5 E, F$ ). Because longer pallidal ISIs are associated with higher firing probability in DLM neurons, this response pattern in pallidal neurons might evoke increased firing in DLM.
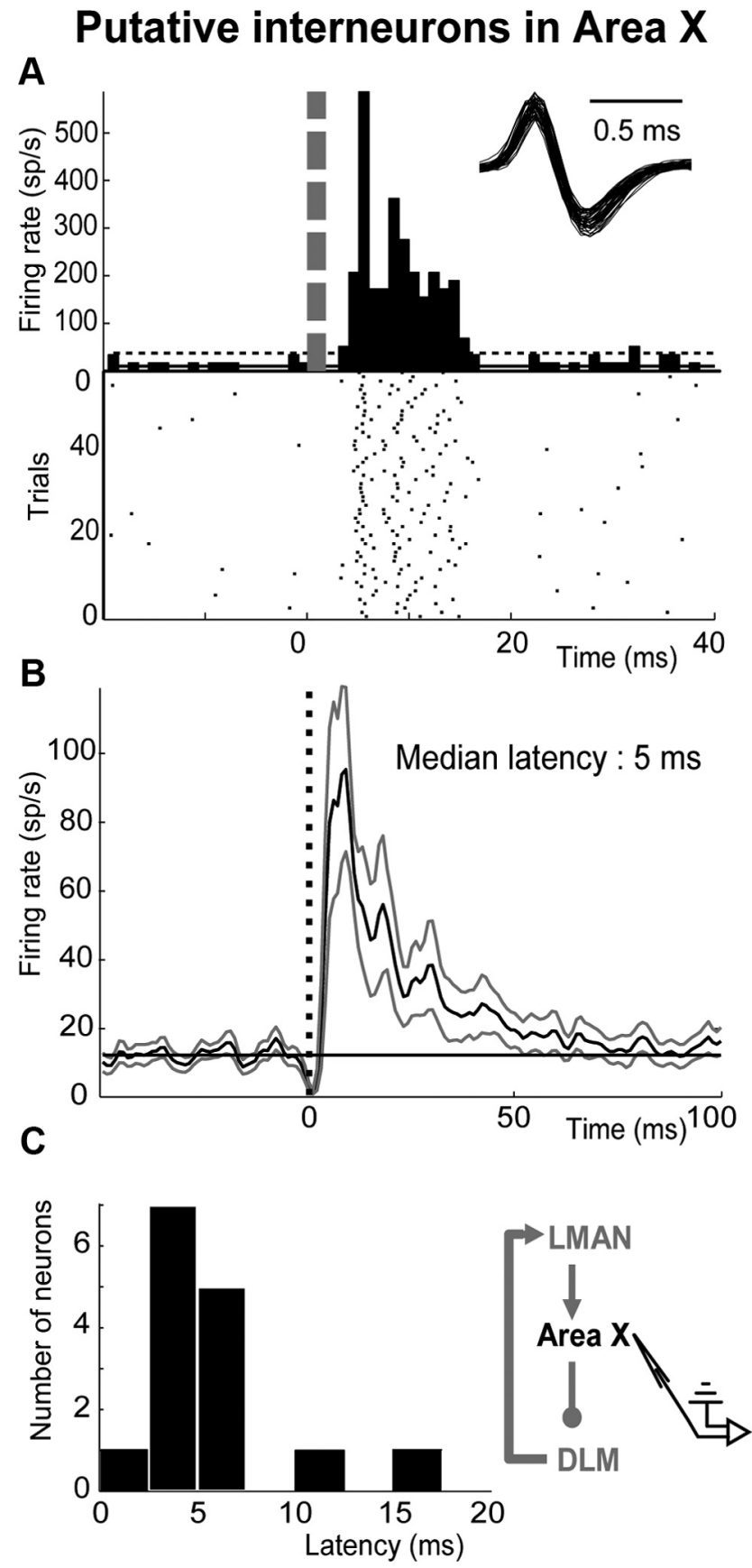

Figure 7. Responses of putative Area X interneuron to HVC electrical stimulation. A, A putative interneuron displaying short-latency response to HVC stimulation consisting of a highfrequency burst of two to four spikes. PSTH of the response aligned to HVC stimulation (top; same conventions as in Fig. $3 A$ ) and raster plot (bottom; 56 trials). Top right inset, Fifty randomly chosen spike waveforms superimposed. $\boldsymbol{B}$, Average PSTH aligned to HVC stimulation over all putative interneurons ( $n=14$; black line indicates the mean, and gray lines indicate mean \pm SEM). C, Distribution of response latencies to HVC stimulation in Area X putative interneurons.

Although the exact relationship between the responses to HVC stimulation evoked in a pallidal cell and its target thalamic neuron could not be investigated here, some relationships can be inferred. On one hand, fast responses in DLM neurons, which have latencies shorter than the average pallidal ISI, might only involve the lengthening of a single pallidal ISI and therefore most likely rely on early inhibitory responses in pallidal neurons. On the other hand, slower responses in DLM neurons could be asso- 


\section{Injection in Area $X$, recording in Area $X$}

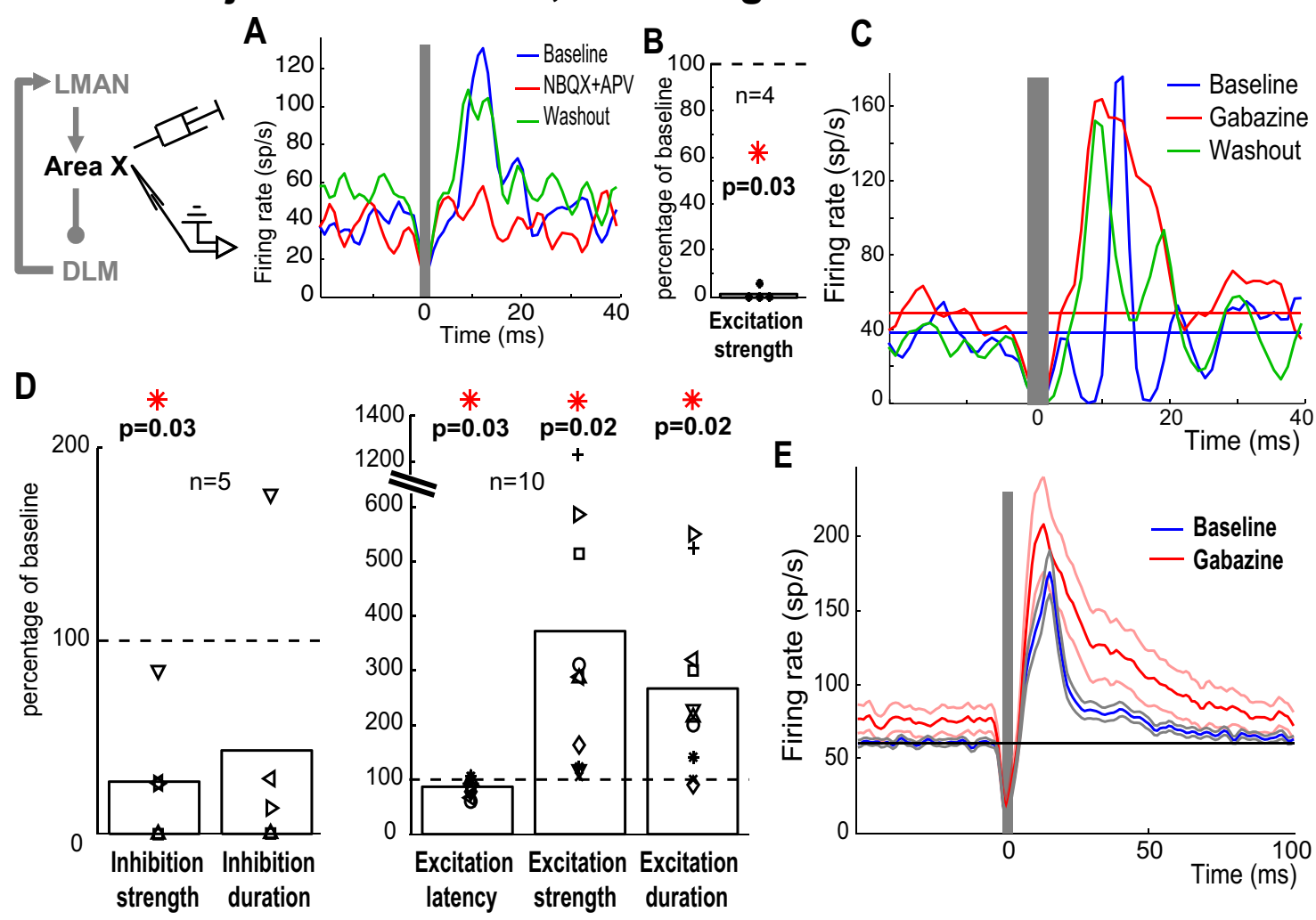

Figure 8. Direct excitation from HVC in Area Xneurons is mediated by glutamate, whereas feedforward inhibition of pallidal neurons is mediated by GABA $A_{A}$ receptors and shapes direct excitation. $A$, Glutamate receptors mediate responses of Area X neurons to HVC electrical stimulation. Top, Smoothed PSTHs (1 ms Gaussian kernel, 1 ms bin) of the responses of a pallidal neuron to HVC stimulation (at $t=0$ ) before (blue), $0-10 \mathrm{~min}$ after (red), and 20 -30 min after (green) injection of NBQX and APV. B, Decrease of excitation strength after NBQX and APV injection with respect to baseline (bar, population averages; circles, single cells; $n=4$ ). $C$, Example of changes in response to HVC stimulation (at $t=0$ ) after gabazine infusion into Area X for a pallidal neuron showing early and late inhibitory components: smoothed PSTHs (1 ms Gaussian kernel, $1 \mathrm{~ms}$ bin) of the response to HVC stimulation during baseline (blue), after injection of gabazine (red), and after washout (green). Although inhibitory components of the response were suppressed by gabazine, the excitatory component had shorter latency and became stronger and longer in duration. $\boldsymbol{D}$, Effect of gabazine on the inhibitory and excitatory components in the response of pallidal neurons to HVC stimulation. From left to right, Strength and duration of inhibitory component $(n=5)$, latency, strength, and duration of excitatory components $(n=10)$ after gabazine injection with respect to baseline. Bars show population averages, and each neuron is represented by a different symbol. After gabazine injection in Area X, inhibitory components of the responses to HVC stimulation were reduced. The excitatory components had shorter latencies and became stronger and longer. The strength and duration of excitatory and inhibitory components of the responses came back to baseline values after washout in most neurons. ${ }^{*} p<0.05$ in all figures. $\boldsymbol{E}$, Average PSTH around HVC stimulation (at $t=0$ ) for all neurons recorded before drug injection (dark blue line), with confidence intervals (light blue lines, mean \pm SEM) and after gabazine injection (red line; confidence intervals in light red, mean \pm SEM). Horizontal black line depicts average baseline spontaneous firing rate. Just as for single neuron responses, the population response to HVC stimulation was stronger and longer during blockade of $\mathrm{GABA}_{\mathrm{A}}$ receptors.

ciated with more complex pallidal response patterns. As suggested by Person and Perkel (2007), fast deceleration associated with an excitation-inhibition response profile could activate thalamic neurons and may thus underlie at least some of the slower responses in DLM neurons. Finally, late excitatory components of pallidal responses might be involved in the truncation of DLM responses, which could serve to maintain or improve timing precision.

Together, our results indicate that Area $\mathrm{X}$ pallidal neurons often display a fast inhibition in response to HVC stimulation, and this short inhibitory response would be expected to disinhibit a target DLM neuron and increase its firing probability.

\section{Putative Area X interneurons exhibit short-latency responses to HVC stimulation}

The spontaneously active pallidal neurons constitute only a minority of Area X neurons. The vast majority of neurons within Area X are striatal spiny neurons, which are homologous to mammalian medium spiny neurons (Farries et al., 2002; Reiner et al., 2004a) but do not project outside of Area X.
Other interneuron types within Area X include fast spiking inhibitory interneurons and cholinergic interneurons (Farries et al., 2002), which, in mammals, display spontaneous activities of $0-10 \mathrm{~Hz}$ (Mallet et al., 2006) and 2-10 Hz (Aosaki et al., 1994), respectively. Area X interneuron populations are generally thought to display little or no spontaneous activity (Farries and Perkel, 2002), which distinguishes them from the only known projection neuron population of Area X, the pallidal neurons. We recorded the response to HVC electrical stimulation in 15 neurons with spontaneous firing below 25 $\mathrm{sp} / \mathrm{s}$ (mean of $11.5 \pm 7.8 \mathrm{sp} / \mathrm{s}$ ). These cells displayed very rapid responses to HVC stimulation, with most cells displaying latencies shorter than $5 \mathrm{~ms}$ (mean of $5.9 \pm 3.8 \mathrm{~ms}$ ) (Fig. 7). Their response was long in duration $(25 \pm 28 \mathrm{~ms}$ ) and very reliable (response probability of $0.8 \pm 0.3$ ). Overall, Area X putative interneurons, at least some of which are inhibitory and project to pallildal neurons (Farries et al., 2005), display very shortlatency and long-duration responses to HVC stimulation, consistent with a role in conveying fast feedforward inhibition onto pallidal neurons that project to DLM. 


\section{Gabazine injection into Area X, recording in DLM $(n=8)$}
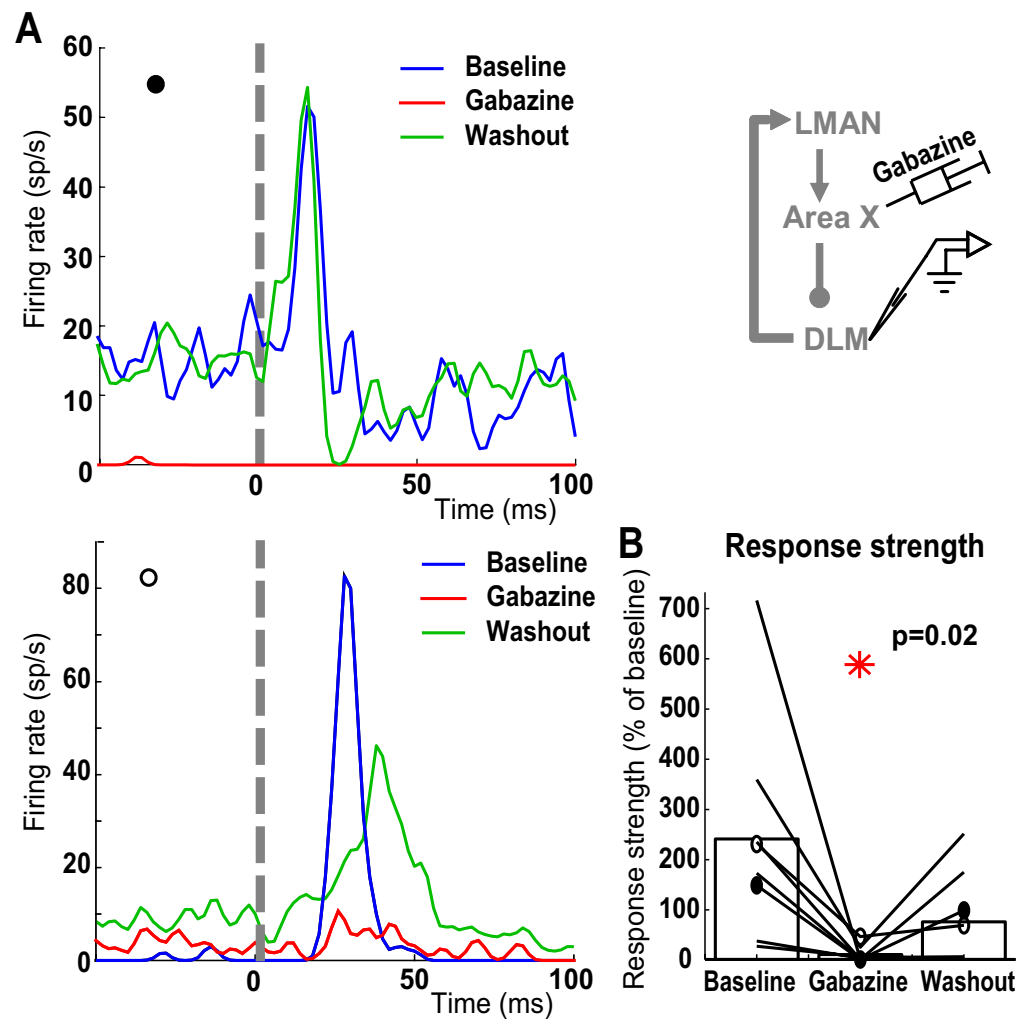

Figure 9. $G A B A_{A}$ receptors in Area $X$ are essential for the response of DLM neurons to HVC electrical stimulation. $A$, Two examples of changes induced by gabazine injection into Area $X$ on the response of DLM neurons to HVC stimulation (dashed lines). The first neuron (top) displayed a significant decrease in spontaneous activity after gabazine injection, whereas the other neuron (bottom) showed no significant change in spontaneous activity over the time course of the experiment. The graphs represent smoothed PSTHs (1 ms Gaussian kernel, $1 \mathrm{~ms}$ bin) of responses before (blue), after gabazine injection (red), and after washout (green). In both cases, the response to HVC stimulation was suppressed by gabazine injection in Area X. B, Effect of gabazine injection on the strength of excitatory responses to HVC stimulation in DLM neurons. Each line represents a single neuron, and bars depict average data. Filled circles represent the first example neuron ( $\boldsymbol{A}$, top), and open circles represent the second one $(\boldsymbol{A}$, bottom). Gabazine injection in Area X suppressed responses to HVC stimulation in all DLM neurons.

suppressed when GABAergic transmission is blocked in Area X. In pallidal cells, injection of gabazine, a reversible $\mathrm{GABA}_{\mathrm{A}}$ receptor blocker, into Area $\mathrm{X}$ had several effects. First, drug application significantly increased spontaneous activity of the recorded pallidal cells from $48.2 \pm$ 23.8 to $73.9 \pm 36.9 \mathrm{sp} / \mathrm{s}(n=10, p=0.03)$. In addition, the strength of the inhibitory component of the response to HVC stimulation was strongly decreased (from $11 \pm 5$ to $4 \pm 6 \%, p=0.01, n=4$ ) (Fig. $8 C, D)$, and its duration was not significantly changed (from $6 \pm 2$ to $3 \pm 6 \mathrm{~ms}$, $p=0.3$ ). In contrast, the excitatory component was significantly stronger (from $63 \pm 30$ to $206 \pm 158 \%, n=10, p=0.01$ ) and longer in duration (from $13 \pm 6$ to $35 \pm 26 \mathrm{~ms}, p=0.02$ ). Close examination of excitatory response latencies calculated before and after drug infusion indicated that early inhibition, even when it was not visible in the PSTHs summarizing the response of a cell to HVC stimulation, delayed the excitatory response to stimulation in these cells (Fig. 8C,D). As a result, latency of the excitatory component significantly decreased after gabazine injection (from $12.4 \pm 3.9$ to $10.9 \pm 4.4$ $\mathrm{ms}, p=0.03)$. This was also apparent in average PSTHs of all cells recorded after injection of gabazine into Area $\mathrm{X}$ aligned to HVC stimulation (Fig. $8 E$ ), showing that early inhibition delays the population response among pallidal cells to stimulation, whereas late inhibition shortens the response.

These results indicate that the local inhibitory network in Area X mediates feedforward inhibition onto pallidal neurons. In half $(48.8 \%)$ of the cells displaying in-

\section{Area X responses to $\mathrm{HVC}$ stimulation are mediated by} glutamatergic input

To confirm that the responses to HVC stimulation in Area X neurons are mediated by the known glutamatergic afferents from HVC (Farries et al., 2005), we injected the glutamate receptor blockers NBQX and APV into Area X. Infusion of these drugs completely suppressed all Area X neuron responses to HVC stimulation (Fig. $8 A, B$ ), consistent with responses in Area $\mathrm{X}$ being mediated by direct excitatory projection from HVC to Area X neurons. Response strength (see Materials and Methods) was decreased by $98.5 \pm 3.0 \%$ ( $130 \pm 91$ vs $2 \pm 3 \%$ of baseline, $n=4$, $p=0.03)$ after drug infusion. In contrast, responses to HVC stimulation in Area X cells did not change after saline injection in Area X (response strength from $56 \pm 27$ to $52 \pm 38 \%, n=3, p=$ 0.9 ). We therefore confirm, as suggested by Farries et al. (2005), that excitatory afferents from HVC drive fast excitation in Area X neurons.

\section{Local inhibition in Area $\mathrm{X}$ mediates fast inhibition of} pallidal neurons

If inhibitory responses to HVC stimulation in pallidal neurons are mediated by Area X GABAergic interneurons, they should be hibition, feedforward inhibition arrived before direct excitation and could create sufficiently long ISIs in the input to DLM neurons to enable them to fire.

\section{Local inhibition in Area $\mathrm{X}$ is necessary for DLM responses}

If the short-latency response in DLM to HVC stimulation is evoked via a fast inhibition of the Area X pallidal neurons, it should be suppressed when GABAergic transmission is blocked in Area X. To test this hypothesis, we recorded DLM neuron responses to HVC stimulation while pharmacologically manipulating neurotransmission in Area X. Injection of the $\mathrm{GABA}_{\mathrm{A}}$ receptor blocker gabazine into Area $\mathrm{X}$ caused no consistent change in DLM neuron spontaneous firing rate $(5 \pm 5$ to $2 \pm 2 \mathrm{sp} / \mathrm{s}, n=$ $8, p=0.2)$. However, the response of all DLM neurons to HVC stimulation was suppressed by gabazine injection in Area $\mathrm{X}$, and response strength decreased by $93.4 \pm 8.8 \%(240 \pm 180$ vs $17 \pm$ $20 \%, n=8, p=0.02$ ) (Fig. $9 A, B$ ). In contrast, after saline injection in Area X, DLM responses to HVC stimulation remained unchanged (response strength, $330 \pm 220$ vs $330 \pm 220 \%, n=3$, $p=0.9$ ). The local inhibitory network in Area $\mathrm{X}$ is thus essential for the response of thalamic neurons to HVC stimulation. 
Glutamatergic transmission to the thalamus is not necessary for DLM responses

Our results suggest that the response of DLM neurons is mainly driven by changes in their GABAergic input from pallidal cells. Alternatively, fast transmission between HVC and DLM could be mediated through a polysynaptic excitatory pathway between these structures. Previous anatomical studies have shown that RA, which receives direct excitatory input from HVC (Kubota and Saito, 1991; Mooney, 1992; Stark and Perkel, 1999), projects to DLM (Wild, 1993; Foster et al., 1997; Vates et al., 1997). Furthermore, DLM neurons show AMPA receptormediated EPSPs (Luo and Perkel, 1999b). Although the RA-to-DLM connection appears anatomically to be much weaker than the primary connections of the song system (Vates et al., 1997 ), it is a putative candidate for mediating fast transmission between HVC and DLM.

We tested whether glutamatergic transmission mediates fast responses in DLM by injecting the AMPA receptor blocker NBQX and the NMDA receptor blocker APV. The spontaneous firing rate was significantly decreased in the presence of these drugs $(3.6 \pm 3.6$ to $1.9 \pm 2.5 \mathrm{sp} / \mathrm{s}$, $n=7, p=0.03$ ) (Fig. 10A). In most DLM neurons, however, the response to HVC stimulation was not affected (Fig. $10 B, C$ ). The response strength was not significantly changed $(140 \pm 120$ vs $90 \pm 80 \%$, $n=7, p=0.4)$, nor was the response latency $(28 \pm 22$ to $27 \pm 23 \mathrm{~ms}, p=0.7)$. Moreover, when the analysis was restricted to neurons displaying a significant change in spontaneous firing rate after application of glutamate receptor blockers, similar results were obtained. Injection of vehicle solution into DLM (0.9\% saline with $0.2 \%$ DMSO and $0.5 \%$ Alexafluor 488) did not modify either spontaneous activity $(1.2 \pm 0.8$ to $1.2 \pm 0.9 \mathrm{sp} / \mathrm{s}, n=3, p=0.9$ ) or responses to HVC stimulation (response strength, $220 \pm 20$ vs $200 \pm 60 \%, p=0.6$ ), ruling out the possibility that our vehicle solution had any effect on DLM neurons. In summary, responses evoked in DLM by HVC stimulation persisted when glutamatergic transmission was blocked in DLM but were altered by manipulations in Area X, which makes a GABAergic projection to DLM.

\section{Very short-latency probabilistic responses to HVC} stimulation in a simple DLM neuron model

As shown above, DLM responses to HVC stimulation are mediated by fast disinhibition through Area X. Because these responses do not rely on excitatory inputs to DLM, they most probably involve postinhibitory rebound in the thalamic neurons. However, the response latency to HVC stimulation is sometimes as short as the rebound latency extrapolated from in vitro data (Person and Perkel, 2005). How can the rebound process not add an additional delay? We investigated the mechanisms of rebound-driven responses in the thalamic neurons using a

\section{NBQX+APV in DLM}
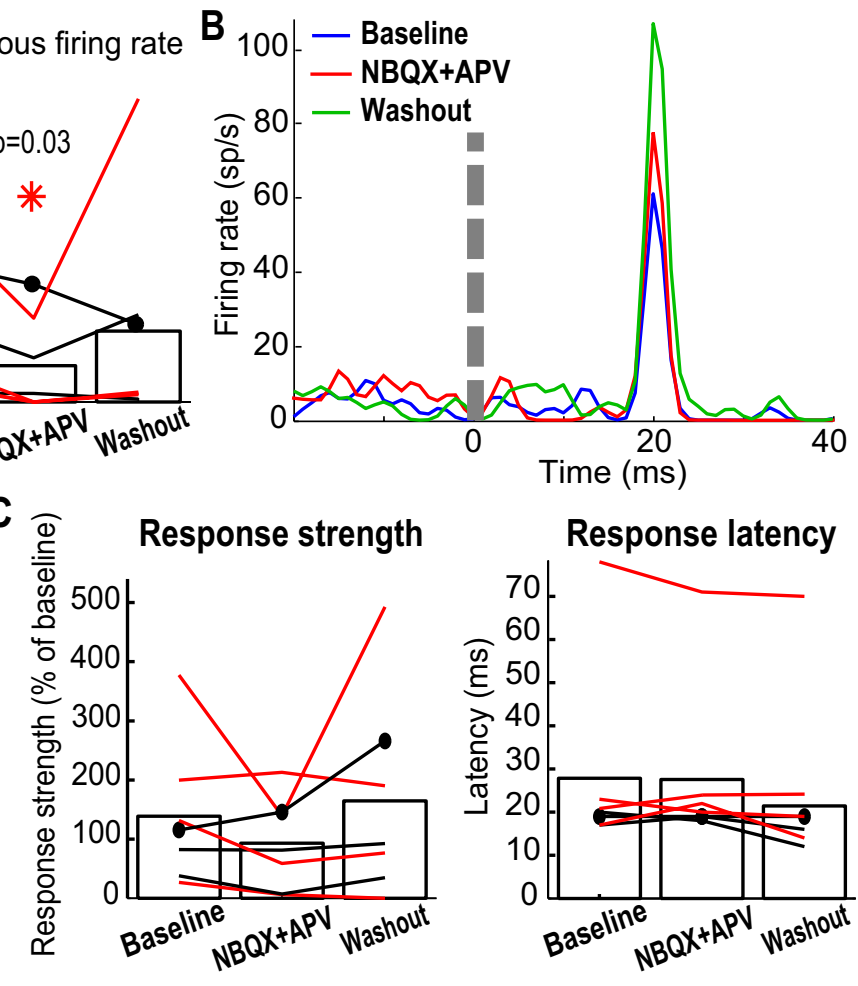

Figure 10. Glutamate receptors are not essential for the response of DLM neurons to HVC electrical stimulation. $\boldsymbol{A}$, Spontaneous activity in DLM was significantly decreased by injection of NBQX and APV into DLM. In each panel in $\boldsymbol{A}$ and $\boldsymbol{C}$, each line represents a after washout (green) of NBQX. The response was not affected by NBOX and APV. C, Population data for changes in response and after washout. Each line represents a single neuron, and bars depict average data. Filled circles represent the example neuron (A). Responses to HVC stimulation were not significantly changed by NBQX and APV.

conductance-based model of a DLM neuron. In this model, we generated theoretical responses to the pallidal spike trains recorded in response to HVC stimulation in 23 randomly selected pallidal neurons displaying an inhibitory response component (Fig. $11 D$ ). The spike trains drove an inhibitory synaptic conductance in a model DLM neuron. We measured model DLM neuron spike times and analyzed the responses relative to the times of HVC stimulation with the same analyses applied to experimentally recorded spike trains. In 17 of 23 cases (representing 23 unique pallidal trains), we found a significant increase in DLM model neuron activity after HVC stimulation, and the distribution of the response latencies was very similar to that experimentally recorded in DLM neurons. In particular, the distribution was dominated by short-latency responses (mean of $24.6 \pm 32.3$ $\mathrm{ms}$, median of $14 \mathrm{~ms}$ ) (Fig. $11 \mathrm{E}$ ). Moreover, like the recorded neurons, the probability of a response among the simulated DLM neurons was low, ranging from 0.04 to 0.9 , averaging $0.25 \pm 0.29$. This simple test reveals that the HVC-evoked inhibition in Area X pallidal cells could evoke rebound firing of suitable probability and latency in a typical DLM neuron.

\section{Discussion}

We report here very fast signal propagation through an avian cortical-BG-thalamo-cortical pathway, with overall transmission delays as short as $10 \mathrm{~ms}$. A rapid disinhibitory process me- 


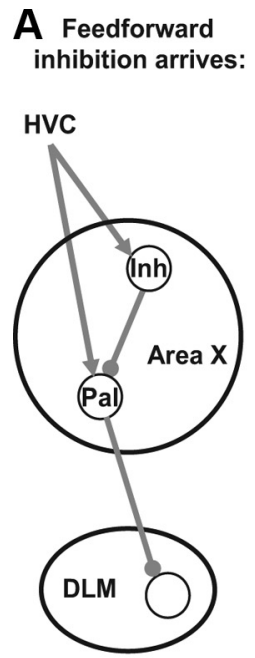

B early in pallidal ISI

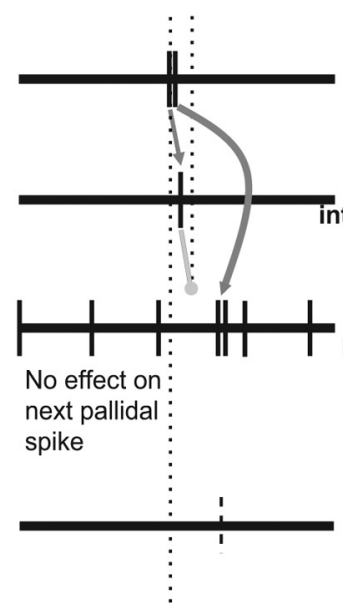

No response in DLM

\section{C late in pallidal ISI}

HVC

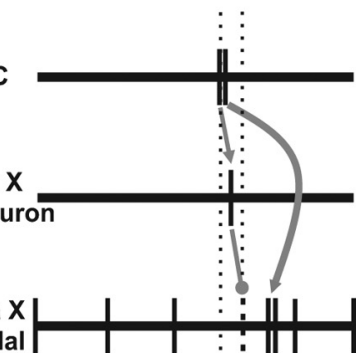

Suppression or : delay of the next pallidal spike

DLM

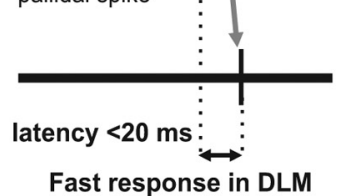

$\mathrm{E}$

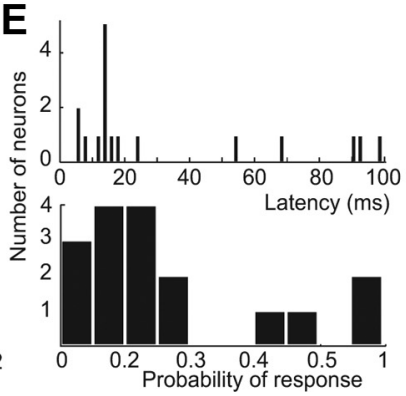

Figure 11. Working model of information transmission between HVC and DLM. $A$, The neural network inside the AFP: HVC sends excitatory input into Area $X$, contacting both pallidal output neurons and inhibitory interneurons, which themselves inhibit pallidal neurons. Area $X$ output neurons send an inhibitory projection to DLM cells. $B$, C, Spikes in a neuron in HVC (top line), an interneuron (second line from top), and a pallidal neuron (third line from top) in Area X, and a DLM neuron (bottom line) in two different configurations. When the feedforward inhibition mediated by Area $X$ interneurons arrives at the pallidal neuron just after it fires a spontaneous spike $(\boldsymbol{B})$, that input is not sufficient to delay the next spike from that pallidal cell or might even cause an advance of pallidal firing by feedforward excitation from HVC. In this situation, the DLM neuron does not fire. In contrast, when the feedforward inhibition arrives later in the pallidal ISI, it delays the next pallidal spike (C). This delays the veto spike that would have prevented the DLM neuron from firing and can thus lead to short-latency firing in DLM. D, Membrane potential in a conductancebased model of a DLM neuron in response to an inhibitory spike train recorded from a real pallidal cell firing spontaneously and in response to HVC stimulation (top raster). $\boldsymbol{E}$, Distribution of latency (top) and probability (bottom) of response in the DLM model neuron, in response to presynaptic spike trains obtained experimentally from 26 different pallidal neurons. Latency denotes the time between HVC stimulation (in the pallidal recording) and the DLM model neuron response and was calculated with the same algorithm used for recorded spike trains (see Fig. 2D).

diates this fast propagation; through feedforward inhibition, ISIs of tonically active pallidal neurons lengthen slightly, transiently interrupting the sustained inhibition of thalamic neurons and greatly increasing their firing probability. Large-diameter axons projecting from pallidal neurons to the thalamus may contribute to the speed of this process. Finally, thalamic responses do not depend on glutamatergic transmission and thus most likely involve postinhibitory rebound. These data reveal a new view of $B G$ transmission whereby pallidal neurons continually send veto signals that arrive just in time to prevent postsynaptic firing. Omission or even slight delay of that inhibitory input triggers a nearly immediate thalamic response.

\section{Mechanism of fast response in DLM}

The main input to the thalamic nucleus DLM is GABAergic and consists of large calyx-like terminals of Area X pallidal neurons onto the DLM soma (Okuhata and Saito, 1987; Vates et al., 1997; Luo and Perkel, 1999a). In vitro, pallidal IPSPs strongly hyperpolarize DLM neurons, which then generate rebound action potentials with a
20-40 ms latency (Luo and Perkel, 1999b; Person and Perkel, 2005). This latency could be shorter in vivo, where spikes in DLM neurons are consistently preceded by $10-20 \mathrm{~ms}$ intervals of silence of the inhibitory presynaptic terminals (Person and Perkel, 2007). These studies suggested that postinhibitory rebound mediates DLM firing in vivo but stopped short of pharmacological manipulation to test the contribution of pallidal firing to DLM activity.

Here, we provide evidence that feedforward GABAergic inhibition triggered by HVC input onto pallidal neurons is necessary and sufficient to trigger a DLM response. In the absence of HVC input, the rapid and regular spontaneous activity of pallidal neurons induces IPSPs separated by $10-25 \mathrm{~ms}$ intervals, imposing a constant veto on DLM neuron firing. Presumably, this inhibition also hyperpolarizes the membrane potential to voltages that ensure the availability of low-threshold calcium channels that support postinhibitory rebound.

A synchronized drive from HVC triggers fast feedforward inhibition of pallidal neurons in Area X. If the feedforward inhibition arrives shortly after the last pallidal spike, it will have little effect (Fig. $11 \mathrm{~B})$. If that inhibition arrives later in the pallidal ISI, it will delay the next spike (Fig. 11C) and trigger an almost instantaneous spike in the target DLM neuron. Indeed, DLM neuron membrane potential rises after each presynaptic spike until it eventually reaches threshold unless an IPSP arrives to stop it (Person and Perkel, 2005). Note that, in vivo, the rise in membrane potential might result from a combination of postinhibitory rebound and concurrent excitatory inputs, although the latter appear not to be necessary for DLM responses.

Increased firing rates preceding long ISIs in the presynaptic pallidal terminal might be important for DLM neuron firing (Person and Perkel, 2007; Kojima and Doupe, 2009). Consistent with intracellular recordings (Person and Perkel, 2005), we propose that increases in pallidal firing rate, such as those observed during song playback, shorten the delay to DLM rebound spikes and increase the firing probability in response to a given presynaptic ISI. However, our results show that such an increase in activity preceding long ISIs is neither necessary nor sufficient to evoke DLM responses, pointing to a critical role for BG feedforward inhibition.

Although we have shown that short pauses $(\sim 20-50 \mathrm{~ms})$ in pallidal activity allow fast thalamic responses, longer inactivation of pallidal neurons (more than seconds) also leads to increased spontaneous thalamic activity (Kojima and Doupe, 2009). Whether this "ungated" thalamic activity relies on excitatory synaptic drive or on DLM neuron excitability remains to be determined.

Based on our results, information propagating through the AFP might reach RA faster than was anticipated previously 
(Kimpo et al., 2003). In that study, peaks of correlated activity between HVC and LMAN led to estimated propagation latencies of $\sim 60 \mathrm{~ms}$. However, many paired recordings showed only a single broad correlation peak, possibly reflecting faster transmission through the AFP. Our approach using electrical stimulation reduces ambiguity in the signal start time and led to our observation of much faster propagation speeds than observed previously.

\section{Relation to function}

BG circuits in general and the AFP in particular are proposed to introduce variability necessary for exploration during motor learning (Graybiel, 2005; Kao et al., 2005; Olveczky et al., 2005). Where within the neural circuit does this variability arise? LMAN neuron spontaneous activity is very irregular, suggesting one source. Moreover, we show here that the response of DLM neurons to activation of cortical areas is highly unreliable. The success or failure of a response, determined by the relative timing of the HVC input to the preceding pallidal spike (Fig. 11), could introduce variability in LMAN response timing. Indeed, assuming LMAN neurons receive convergent input from many DLM neurons, they may be activated with different latencies depending on which population of presynaptic neurons is active.

In addition to introducing variability, the AFP may provide patterned signals to guide changes in motor output (Kao et al., 2008). Such signals would most likely come from HVC and be transformed in the AFP, potentially through dopaminedependent mechanisms. Indeed, dopamine could alter information flow through Area X depending on the social context (Sasaki et al., 2006) through short- or long-term synaptic effects (Ding and Perkel, 2004). In this view, an overlap in RA with the "production signal" sent through the monosynaptic HVC-RA pathway would provide the AFP signal with a simple mechanism to alter online behavior. Because song-related signals are very short (10-20 ms) (Hahnloser et al., 2002; Kozhevnikov and Fee, 2007), there is not much time for transmission through the AFP. Our results suggest that latencies through the AFP might be shorter than $20 \mathrm{~ms}$ and would thus allow interaction of motor pathway and AFP signals within RA.

\section{Comparison with mammalian BG}

Area X differs from mammalian BG in its gross anatomical structure but displays similar circuitry at a finer scale. Indeed, Area X comprises primarily striatal neurons, but, consistent with mammalian circuitry, it is its pallidal component that directly projects to the thalamus (Bottjer et al., 1989; Luo and Perkel, 1999a; Farries and Perkel, 2002; Carrillo and Doupe, 2004; Reiner at al., 2004a). It contains the anatomical substrate for a disinhibitory pathway homologous to the direct pathway through mammalian BG (Reiner et al., 2004a; Farries et al., 2005), which, as shown here, is functionally critical. Indeed, afferents from the cortical structure HVC drive feedforward inhibition in BG output through striatal neurons and disinhibit thalamic target neurons. Interestingly, avian striatal inhibitory neurons may require high synchronization of their cortical inputs to drive such feedforward inhibition, similar to striatal medium spiny neurons (Charpier et al., 1999). In contrast to mammalian pallidal neurons, Area X output neurons also receive monosynaptic input from cortical glutamatergic afferents (Farries et al., 2005). This input provides a powerful and sensitive drive to pallidal neurons, with broadly distributed latencies, consistent with previous estimations of transmission delays from HVC to Area X (Hahnloser et al., 2006). This connection might provide Area X with a pathway functionally equivalent to the "hyperdirect" pathway through the subthalamic nucleus in mammals
(Nambu et al., 2000), which is not connected with Area X (Person et al., 2008). This analog of the hyperdirect pathway may increase the selectivity of disinhibitory signals transmitted through the direct pathway both spatially, by broadly inhibiting DLM during focused disinhibition, and temporally, by limiting the duration of the disinhibitory signal (Nambu et al., 2000).

\section{Speed specialization}

The AFP may be an example of a BG circuit specialized for rapid processing of cortical inputs. First, BG output axons have very large diameters $(3 \mu \mathrm{m})$ for vertebrate CNS neurons, especially compared with their mammalian counterparts $(<1 \mu \mathrm{m}$ in rats) (Bodor et al., 2008) or with other axons in the zebra finch brain $(<1 \mu \mathrm{m})$, allowing fast conduction. Second, in contrast with the mammalian BG motor loop, in which transmission along the direct pathway is slower than feedforward excitation through the subthalamic nucleus (Nambu et al., 2000), feedforward inhibition is surprisingly fast in the song-related BG. Indeed, it arrives fast enough to prevent or slow the rise of monosynaptic excitation from cortical structures. This rapid feedforward inhibition is mediated by GABAergic Area X neurons, most likely interneurons. In particular, it might involve the fast-spiking interneurons, which provide fast feedforward inhibition in the mammalian striatum (Mallet et al., 2006) and are present in Area X (Farries et al., 2002; Reiner et al., 2004a).

The mammalian pallido-thalamic connection displays powerful GABAergic synapses similar to those seen in songbirds (Bodor et al., 2008; Wanaverbecq et al., 2008); the cellular mechanisms described here may well be at least partially available in mammals. Whereas motor output often operates on longer timescales (>100 ms) in mammals, specific BG systems involved in fast sensorimotor tasks such as speech in humans or whisking in rats are subjected to similar constraints as the AFP. Although information propagation has not yet been carefully characterized in these circuits, they may display similar specializations for fast transmission as those presented here.

\section{References}

Aosaki T, Tsubokawa H, Ishida A, Watanabe K, Graybiel AM, Kimura M (1994) Responses of tonically active neurons in the primate's striatum undergo systematic changes during behavioral sensorimotor conditioning. J Neurosci 14:3969-3984.

Bagshaw EV, Evans MH (1976) Measurement of current spread from microelectrodes when stimulating within the nervous system. Exp Brain Res 25:391-400.

Baker SN, Gerstein GL (2001) Determination of response latency and its application to normalization of cross-correlation measures. Neurol Comput 13:1351-1377.

Bodor AL, Giber K, Rovó Z, Ulbert I, Acsády L (2008) Structural correlates of efficient GABAergic transmission in the basal ganglia-thalamus pathway. J Neurosci 28:3090-3102.

Bottjer SW, Miesner EA, Arnold AP (1984) Forebrain lesions disrupt development but not maintenance of song in passerine birds. Science 224:901-903.

Bottjer SW, Halsema KA, Brown SA, Miesner EA (1989) Axonal connections of a forebrain nucleus involved with vocal learning in zebra finches. J Comp Neurol 279:312-326.

Broussard DM, Brontë-Stewart HM, Lisberger SG (1992) Expression of motor learning in the response of the primate vestibuloocular reflex pathway to electrical stimulation. J Neurophysiol 67:1493-1508.

Carrillo GD, Doupe AJ (2004) Is the songbird Area X striatal, pallidal, or both? An anatomical study. J Comp Neurol 473:415-437.

Charpier S, Mahon S, Deniau JM (1999) In vivo induction of striatal longterm potentiation by low-frequency stimulation of the cerebral cortex. Neuroscience 91:1209-1222.

Deniau JM, Chevalier G (1985) Disinhibition as a basic process in the expression of striatal functions. II. The striato-nigral influence on thalamocortical cells of the ventromedial thalamic nucleus. Brain Res 334:227-233. 
Destexhe A, Bal T, McCormick DA, Sejnowski TJ (1996) Ionic mechanisms underlying synchronized oscillations and propagating waves in a model of ferret thalamic slices. J Neurophysiol 76:2049-2070.

Ding L, Perkel DJ (2004) Long-term potentiation in an avian basal ganglia nucleus essential for vocal learning. J Neurosci 24:488-494.

Farries MA, Perkel DJ (2002) A telencephalic nucleus essential for song learning contains neurons with physiological characteristics of both striatum and globus pallidus. J Neurosci 22:3776-3787.

Farries MA, Ding L, PerkelDJ (2005) Evidence for "direct" and "indirect" pathways through the song system basal ganglia. J Comp Neurol 484:93-104.

Follett KA, Mann MD (1986) Effective stimulation distance for current from macroelectrodes. Exp Neurol 92:75-91.

Foster EF, Mehta RP, Bottjer SW (1997) Axonal connections of the medial magnocellular nucleus of the anterior neostriatum in zebra finches. J Comp Neurol 382:364-381.

Gale SD, Person AL, Perkel DJ (2008) A novel basal ganglia pathway forms a loop linking a vocal learning circuit with its dopaminergic input. J Comp Neurol 508:824-839.

Glaze CM, Troyer TW (2007) Behavioral measurements of a temporally precise motor code for birdsong. J Neurosci 27:7631-7639.

Goller F, Cooper BG (2004) Peripheral motor dynamics of song production in the zebra finch. Ann NY Acad Sci 1016:130-152.

Graybiel AM (2005) The basal ganglia: learning new tricks and loving it. Curr Opin Neurobiol 15:638-644.

Hahnloser RH, Kozhevnikov AA, Fee MS (2002) An ultra-sparse code underlies the generation of neural sequences in a songbird. Nature 419:65-70.

Hahnloser RH, Kozhevnikov AA, Fee MS (2006) Sleep-related neural activity in a premotor and a basal-ganglia pathway of the songbird. J Neurophysiol 96:794-812.

Heil P, Scheich H (1991) Functional organization of the avian auditory cortex analogue. II. Topographic distribution of latency. Brain Res 539: 121-125.

Hines ML (1998) The neurosimulator NEURON. In: Methods in neuronal modeling (Koch C, Segev I, eds), pp 129-136. Cambridge, MA: MIT.

Jarvis ED, Güntürkün O, Bruce L, Csillag A, Karten H, Kuenzel W, Medina L, Paxinos G, Perkel DJ, Shimizu T, Striedter G, Wild JM, Ball GF, DugasFord J, Durand SE, Hough GE, Husband S, Kubikova L, Lee DW, Mello CV, Powers A, Siang C, Smulders TV, Wada K, White SA, Yamamoto K, Yu J, Reiner A, Butler AB (2005) Avian brain nomenclature consortium. Avian brains and a new understanding of vertebrate brain evolution. Nat Rev Neurosci 6:151-159.

Kao MH, Doupe AJ, Brainard MS (2005) Contributions of an avian basal gangliaforebrain circuit to real-time modulation of song. Nature 433:638-643.

Kao MH, Wright BD, Doupe AJ (2008) Neurons in a forebrain nucleus required for vocal plasticity rapidly switch between precise firing and variable bursting depending on social context. J Neurosci 28:13232-13247.

Kimpo RR, Theunissen FE, Doupe AJ (2003) Propagation of correlated activity through multiple stages of a neural circuit. J Neurosci 23:5750-5761.

Kojima S, Doupe AJ (2009) Activity propagation in an avian basal gangliathalamocortical circuit essential for vocal learning. J Neurosci 29:4782- 4793.

Kozhevnikov AA, Fee MS (2007) Singing-related activity of identified HVC neurons in the zebra finch. J Neurophysiol 97:4271-4283.

Kubota M, Saito N (1991) NMDA receptors participate differentially in two different synaptic inputs in neurons of the zebra finch robust nucleus of the archistriatum in vitro. Neurosci Lett 125:107-109.

Liberman AM, Harris KS, Kinney JA, Lane H (1961) The discrimination of relative onset-time of the components of certain speech and nonspeech patterns. J Exp Psychol 61:379-388.

Luo M, Perkel DJ (1999a) Long-range GABAergic projection in a circuit essential for vocal learning. J Comp Neurol 403:68-84.

Luo M, Perkel DJ (1999b) A GABAergic, strongly inhibitory projection to a thalamic nucleus in the zebra finch song system. J Neurosci 19:6700-6711.

Luo M, Perkel DJ (2002) Intrinsic and synaptic properties of neurons in an avian thalamic nucleus during song learning. J Neurophysiol 88:1903- 1914.

MacDougall-Shackleton SA, Hulse SH, Ball GF (1998) Neural correlates of singing behavior in male zebra finches (Taeniopygia guttata). J Neurobiol 36:421-430.

Maggi CA, Meli A (1986) Suitability of urethane anesthesia for physiopharmacological investigations in various systems. I. General considerations. Experentia 42:109-114.

Mallet N, Ballion B, Le Moine C, Gonon F (2006) Cortical inputs and GABA interneurons imbalance projection neurons in the striatum of Parkinsonian rats. J Neurosci 26:3875-3884.
McCormick DA, Huguenard JR (1992) A model of the electrophysiological properties of thalamocortical relay neurons. J Neurophysiol 68:1384-1400.

Mooney R (1992) Synaptic basis for developmental plasticity in a birdsong nucleus. J Neurosci 12:2464-2477.

Mooney R, Konishi M (1991) Two distinct inputs to an avian song nucleus activate different glutamate receptor subtypes on individual neurons. Proc Natl Acad Sci U S A 88:4075-4079.

Nambu A, Tokuno H, Hamada I, Kita H, Imanishi M, Akazawa T, Ikeuchi Y, Hasegawa N (2000) Excitatory cortical inputs to pallidal neurons via the subthalamic nucleus in the monkey. J Neurophysiol 84:289-300.

Nottebohm F, Stokes TM, Leonard CM (1976) Central control of song in the canary, Serinus canarius. J Comp Neurol 165:457-486.

Okuhata S, Saito N (1987) Synaptic connections of thalamo-cerebral vocal nuclei of the canary. Brain Res Bull 18:35-44.

Olveczky BP, Andalman AS, Fee MS (2005) Vocal experimentation in the juvenile songbird requires a basal ganglia circuit. PLoS Biol 3:e153.

Person AL, Perkel DJ (2005) Unitary IPSPs drive precise thalamic spiking in a circuit required for learning. Neuron 46:129-140.

Person AL, Perkel DJ (2007) Pallidal neuron activity increases during sensory relay through thalamus in a songbird circuit essential for learning J Neurosci 27:8687-8698.

Person AL, Gale SD, Farries MA, Perkel DJ (2008) Organization of the songbird basal ganglia, including Area X. J Comp Neurol 508:840-866.

Pollak GD, Burger RM, Klug A (2003) Dissecting the circuitry of the auditory system. Trends Neurosci 26:33-39.

Ranck JB Jr (1975) Which elements are excited in electrical stimulation of mammalian central nervous system: a review. Brain Res 98:417-440.

Reiner A, Laverghetta AV, Meade CA, Cuthbertson SL, Bottjer SW (2004a) An immunohistochemical and pathway tracing study of the striatopallidal organization of Area X in the male zebra finch. J Comp Neurol 469:239-261.

Reiner A, Perkel DJ, Bruce LL, Butler AB, Csillag A, Kuenzel W, Medina L, Paxinos G, Shimizu T, Striedter G, Wild M, Ball GF, Durand S, Güntürkün O, Lee DW, Mello CV, Powers A, White SA, Hough G, Kubikova L, Smulders TV, Wada K, Dugas-Ford J, Husband S, Yamamoto K, Yu J, Siang C, Jarvis ED, Gütürkün O (2004b) Revised nomenclature for avian telencephalon and some related brainstem nuclei. J Comp Neurol 473:377-414.

Sasaki A, Sotnikova TD, Gainetdinov RR, Jarvis ED (2006) Social contextdependent singing-regulated dopamine. J Neurosci 26:9010-9014.

Scharff C, Nottebohm F (1991) A comparative study of the behavioral deficits following lesions of various parts of the zebra finch song system: implications for vocal learning. J Neurosci 11:2896-2913.

Sen K, Theunissen FE, Doupe AJ (2001) Feature analysis of natural sounds in the songbird auditory forebrain. J Neurophysiol 86:1445-1458.

Somogyi P, Hodgson AJ (1985) Antisera to gamma-aminobutyric acid. III. Demonstration of GABA in Golgi-impregnated neurons and in conventional electron microscopic sections of cat striate cortex. J Histochem Cytochem 33:249-257.

Stark LL, Perkel DJ (1999) Two-stage, input-specific synaptic maturation in a nucleus essential for vocal production in the zebra finch. J Neurosci 19:9107-9116.

Tehovnik EJ, Tolias AS, Sultan F, Slocum WM, Logothetis NK (2006) Direct and indirect activation of cortical neurons by electrical microstimulation. J Neurophysiol 96:512-521.

Thomson AM (1988) Inhibitory postsynaptic potentials evoked in thalamic neurons by stimulation of the reticularis nucleus evoke slow spikes in isolated rat brain slices. I. Neuroscience 25:491-502.

Troyer TW, Doupe AJ (2000) An associational model of birdsong sensorimotor learning I. Efference copy and the learning of song syllables. J Neurophysiol 84:1204-1223.

Vates GE, Vicario DS, Nottebohm F (1997) Reafferent thalamo-"cortical" loops in the song system of oscine songbirds. J Comp Neurol 380: 275-290.

Wanaverbecq N, Bodor AL, Bokor H, Slézia A, Lüthi A, Acsády L (2008) Contrasting the functional properties of GABAergic axon terminals with single and multiple synapses in the thalamus. J Neurosci 28:11848-11861.

Wild JM (1993) Descending projections of the songbird nucleus robustus archistriatalis. J Comp Neurol 338:225-241.

Wild JM, Williams MN, Howie GJ, Mooney R (2005) Calcium-binding proteins define interneurons in HVC of the zebra finch (Taeniopygia guttata). J Comp Neurol 483:76-90. 